\title{
OPTIMAL CONTROL PROBLEMS WITH SET-VALUED CONTROL AND STATE CONSTRAINTS*
}

\author{
ZSOLT PÁLES ${ }^{\dagger}$ AND VERA ZEIDAN ${ }^{\ddagger}$
}

\begin{abstract}
In this paper a general optimal control problem with pure state and mixed controlstate constraints is considered. These constraints are of the form of set-inclusions. Second-order necessary optimality conditions for weak local minimum are derived for this problem in terms of the original data. In particular the nonemptiness of the set of critical directions and the evaluation of its support function are expressed in terms of the given functions and set-valued maps. In order that the Lagrange multiplier corresponding to the mixed control-state inclusion constraint be represented via an integrable function, a strong normality condition involving the notion of the critical tangent cone is introduced.
\end{abstract}

Key words. first- and second-order optimality conditions, critical cone, critical tangent cone, set-valued constraints

AMS subject classifications. 49B27, 49B36

DOI. $10.1137 / \mathrm{S} 1052623401389774$

1. Introduction. Consider the following optimization problem:

$$
\text { Minimize } F(z) \text { subject to } G(z) \in \mathbf{Q}, H(z)=0 \text {, }
$$

where $F: \mathcal{D} \rightarrow \mathbb{R}, G: \mathcal{D} \rightarrow X, H: \mathcal{D} \rightarrow Y$, and $X, Y, Z$ are Banach spaces, $\mathcal{D} \subset Z$ is nonempty and open, and $\mathbf{Q} \subset X$ is a closed convex set with nonempty interior.

The prototype of such problems arises, for instance, in optimal control theory with control and/or state constraints in the inclusion form $x(t) \in Q(t)$.

Better understanding of optimality conditions is an ongoing topic of research for several researchers. This question is of great value in theory and in applications. Usually, such conditions must be given in terms of the original data of the problem and, in the context of necessity, are expected to be as strong as they can be.

In 1988, Kawasaki [Kaw88a], [Kaw91] discovered, for the problem $(\mathcal{P})$, where $\mathbf{Q}$ is a cone, second-order necessary conditions that contain an extra term manifesting the presence of infinitely many inequalities in the constraint $G(z) \in \mathbf{Q}$. This phenomenon is known as the "envelope-like effect" and extends the results found in [BT80] and [BTZ82]. Such a result was generalized by Cominetti in [Com90]. Both results assumed a Mangasarian-Fromovitz-type condition.

In [PZ94a] the authors generalized the results of [Kaw88a], [Kaw91], [Kaw92], and [Com90] to the nondifferentiable case without assuming a Mangasarian-Fromovitz condition. The second-order admissible variation set used therein (defined first by Dubovitskii and Milyutin in [DM63] and [DM65]) is described in the following definition.

*Received by the editors May 24, 2001; accepted for publication (in revised form) March 7, 2003; published electronically August 22, 2003.

http://www.siam.org/journals/siopt/14-2/38977.html

$\dagger$ Institute of Mathematics and Informatics, University of Debrecen, H-4010 Debrecen, Pf. 12, Hungary (pales@math.klte.hu). Research supported by the Hungarian Scientific Research Fund (OTKA), grant T-038072, and by the Hungarian Higher Education, Research, and Development Fund (FKFP), grant 0215/2001.

${ }^{\ddagger}$ Department of Mathematics, Michigan State University, East Lansing, MI 48824 (zeidan@math. msu.edu). Research supported partially by the Department of Mathematics at Michigan State University and by the National Science Foundation under grant DMS-0072598. 
Definition. Let $X$ be a normed space, $\mathbf{Q} \subset X, x \in \mathbf{Q}$, and $d \in X$. A vector $v \in X$ is called $a$ second-order admissible variation of $\mathbf{Q}$ at $x$ in the direction $d$ if there exists $\bar{\varepsilon}>0$ such that

$$
x+\varepsilon d+\varepsilon^{2}(v+u) \in \mathbf{Q} \quad \text { for all } 0<\varepsilon<\bar{\varepsilon},\|u\|<\bar{\varepsilon}, u \in X .
$$

The set of all such variations is denoted by $V(x, d \mid \mathbf{Q})$. It follows directly from the definition that $V(x, d \mid \mathbf{Q})$ is an open set. If $\mathbf{Q}$ is also convex, then $V(x, d \mid \mathbf{Q})$ is convex as well.

In order to derive meaningful second-order optimality conditions, it is necessary to select directions $d$ that guarantee the nonemptiness of $V(x, d \mid \mathbf{Q})$. Such directions $d \in X$ are labeled as the critical directions of $\mathbf{Q}$ at $x$ and form a set called the critical direction cone to $\mathbf{Q}$ at $x$. Throughout this paper, this cone will be denoted by $C(x \mid \mathbf{Q})$. It can be easily seen that $C(x \mid \mathbf{Q})$ is a convex cone if $\mathbf{Q}$ is convex.

Define

$$
S(x \mid \mathbf{Q}):=\operatorname{cone}(\mathbf{Q}-x):=\{\lambda(q-x) \mid q \in \mathbf{Q}, \lambda>0\}
$$

and its closure

$$
T(x \mid \mathbf{Q}):=\overline{\operatorname{cone}}(\mathbf{Q}-x)=\operatorname{cl} S(x \mid \mathbf{Q}) .
$$

If $\mathbf{Q}$ is convex, then for the nonemptiness of $V$ it is necessary, but not sufficient, that the interior of $\mathbf{Q}$ be nonempty and that $d$ belong to $T(x \mid \mathbf{Q})$. However, the nonemptiness of $V$ is assured if intr $\mathbf{Q} \neq \emptyset$ and $d \in S(x \mid \mathbf{Q})$. Therefore, for convex $\mathbf{Q}$, we have

$$
S(x \mid \mathbf{Q}) \subset C(x \mid \mathbf{Q}) \subset T(x \mid \mathbf{Q}) .
$$

In the applications, when the inequality-type constraint is expressed in terms of several inclusions and inequalities, it is useful to know the following easy-to-establish product rules:

$$
C(x \mid \mathbf{Q})=\prod_{i=1}^{k} C\left(x_{i} \mid \mathbf{Q}_{i}\right) \quad \text { and } \quad V(x, d \mid \mathbf{Q})=\prod_{i=1}^{k} V\left(x_{i}, d_{i} \mid \mathbf{Q}_{i}\right),
$$

where $\mathbf{Q}_{1}, \ldots, \mathbf{Q}_{k}$ are subsets of vector spaces, $\mathbf{Q}:=\mathbf{Q}_{1} \times \cdots \times \mathbf{Q}_{k}, x=\left(x_{1}, \ldots, x_{k}\right) \in$ $\mathbf{Q}, d=\left(d_{1}, \ldots, d_{k}\right) \in C(x \mid \mathbf{Q})$.

In order to recall the first- and second-order necessary conditions for $(\mathcal{P})$ obtained in [PZ94a, Corollary 2] and in [PZ96], we need to introduce the following notation and notions.

- A point $\widehat{z} \in \mathcal{D}$ is called an admissible point for $(\mathcal{P})$ if $G(\widehat{z}) \in \mathbf{Q}$ and $H(\widehat{z})=0$ hold. A point $\widehat{z} \in \mathcal{D}$ is a solution (local minimum) of the problem if it is admissible and there exists a neighborhood $U$ of $\widehat{z}$ such that $F(z) \geq F(\widehat{z})$ for all admissible points $z \in U$.

- A point $\widehat{z} \in \mathcal{D}$ is called a regular point for $(\mathcal{P})$ if $F, G$, and $H$ are strictly Fréchet differentiable at $\widehat{z}$ and the range of the linear operator $H^{\prime}(\widehat{z})$ is a closed subspace of $Y$.

Let $\widehat{z}$ be an admissible regular point for $(\mathcal{P})$ and $d \in Z$.

- A vector $\delta z \in Z$ is called a critical direction at $\widehat{z}$ for $(\mathcal{P})$ if

$$
F^{\prime}(\widehat{z}) \delta z \leq 0, \quad G^{\prime}(\widehat{z}) \delta z \in C(G(\widehat{z}) \mid \mathbf{Q}), \quad H^{\prime}(\widehat{z}) \delta z=0 .
$$


- A vector $\delta z \in Z$ is called a regular direction at $\widehat{z}$ for $(\mathcal{P})$ if the second-order directional derivative of $L:=(F, G, H)$,

$$
L^{\prime \prime}(\widehat{z}, \delta z):=\lim _{\varepsilon \rightarrow 0+} 2 \frac{L(\widehat{z}+\varepsilon \delta z)-L(\widehat{z})-\varepsilon L^{\prime}(\widehat{z}) \delta z}{\varepsilon^{2}},
$$

exists.

Clearly, the zero vector is always a regular critical direction at $\widehat{z}$ for $(\mathcal{P})$.

Now we are ready to state a particular case of the result of [PZ94a, Corollary 2].

THEOREM 1.1. Let $\widehat{z}$ be a regular local solution of the above problem $(\mathcal{P})$. Then, for all regular critical directions $\delta z$, there correspond Lagrange multipliers $\lambda \geq 0$, $x^{*} \in X^{*}$, and $y^{*} \in Y^{*}$ (which depend on $\delta z$ ) such that at least one of them is different from zero and the following relations hold:

$$
\lambda F^{\prime}(\widehat{z}) z+\left\langle x^{*}, G^{\prime}(\widehat{z}) z\right\rangle+\left\langle y^{*}, H^{\prime}(\widehat{z}) z\right\rangle=0 \quad \text { for } \quad z \in Z,
$$

and

$$
\lambda F^{\prime \prime}(\widehat{z}, \delta z)+\left\langle x^{*}, G^{\prime \prime}(\widehat{z}, \delta z)\right\rangle+\left\langle y^{*}, H^{\prime \prime}(\widehat{z}, \delta z)\right\rangle \geq 2 \delta^{*}\left(x^{*} \mid V\left(G(\widehat{z}), G^{\prime}(\widehat{z}) \delta z \mid \mathbf{Q}\right)\right)
$$

(Here $\delta^{*}$ denotes the support functional defined by $\delta^{*}\left(x^{*} \mid V\right):=\sup _{v \in V}\left\langle x^{*}, v\right\rangle$ for $\left(x^{*} \in X^{*}\right)$, and $N(x \mid \mathbf{Q})$ denotes the adjoint cone of $T(x \mid \mathbf{Q})$, that is, the cone of outward normals to the set $\mathbf{Q}$ at the point $x$.)

As we have seen, the criticality of $\delta z$ requires that $d \in C(x \mid \mathbf{Q})$, where $x:=G(\widehat{z})$ and $d:=G^{\prime}(\widehat{z}) \delta z$. However, in order that $d$ be in $C(x \mid \mathbf{Q})$, it is only necessary that $\mathbf{Q}$ have a nonempty interior and that $d$ belong to $T(x \mid \mathbf{Q})$. If $d \in S(x \mid \mathbf{Q})$, then $V(x, d \mid \mathbf{Q})$ is nonempty and $V(x, d \mid \mathbf{Q})=\operatorname{cone}(\operatorname{cone}(\operatorname{intr} \mathbf{Q}-x)-d)$ (cf. [PZ94a, Theorem 4]). In this case the right-hand side in the second-order condition (1.3) vanishes. However, examples are provided by Kawasaki [Kaw88a] which show that the necessary conditions with extra term, that is, when $d \in T(x \mid \mathbf{Q})$, handle situations that cannot be handled with previous results where $d$ is taken from $S(x \mid \mathbf{Q})$. Thus, one has to also consider directions $d \in T(x \mid \mathbf{Q}) \backslash \operatorname{cone}(\mathbf{Q}-x)$. In this important case two questions naturally arise from Theorem 1.1:

(i) How can we check the nonemptiness of $V(x, d \mid \mathbf{Q})$; that is, how can the critical cone $C(x \mid \mathbf{Q})$ be characterized in terms of $\mathbf{Q}$ ?

(ii) How can we evaluate the support function of $V(x, d \mid \mathbf{Q})$ ?

A significant setting is the case when $\mathbf{Q}$ is a subset of $\mathcal{C}\left(T, \mathbb{R}^{\kappa}\right)$ defined by

$$
\mathbf{Q}=\operatorname{sel}_{C}(Q):=\left\{x \in \mathcal{C}\left(T, \mathbb{R}^{\kappa}\right) \mid x(t) \in Q(t) \text { for all } t \in T\right\},
$$

where $Q$ is a lower semicontinuous set-valued map whose images are closed, convex sets with nonempty interior, and $T$ is a compact Hausdorff space. The importance of this type of constraint stems from control problems with state constraints.

Another case of interest is when $\mathbf{Q}$ is a subset of $\mathcal{L}^{\infty}\left(\Omega, \mathbb{R}^{\gamma}\right)$ defined by

$$
\mathbf{Q}=\operatorname{sel}_{\infty}(Q):=\left\{x \in \mathcal{L}^{\infty}\left(\Omega, \mathbb{R}^{\gamma}\right) \mid x(t) \in Q(t) \text { for a.e. } t \in \Omega\right\},
$$

where $Q$ is a measurable set-valued map whose images are closed and have nonempty interior, and $(\Omega, \mathcal{A}, \nu)$ is a complete finite measure space. This type of constraint is typical for control constraints in control problems. 
The main goal of this paper is to investigate these two types of constraints so that the application of Theorem 1.1 to optimal control problems leads to weak-local optimality necessary conditions that are phrased in terms of the original data; part of these results is announced in [PZ01]. However, given the fact that the state variable $x$ and the control variable $u$ belong to different spaces, it has been known for a long time (see, e.g., [PZ94b, Theorem 3]) that to obtain a result for an optimal control problem by applying an abstract result like Theorem 1.1, one should first derive a specialized version of that abstract result that takes into account the distinct features of each of these variables. Such a result has been developed in [PZ94b] and will be recalled in the next section.

Since, for control problems, the constraint set $\mathbf{Q}$ could be a product of different types of constraints, that is, endpoint set-inclusion, control and state set-inclusion, therefore, we shall need the following sum rule for the extra term in (1.3):

$$
\delta^{*}\left(x^{*} \mid V(x, d \mid \mathbf{Q})\right)=\sum_{i=1}^{k} \delta^{*}\left(x_{i}^{*} \mid V\left(x_{i}, d_{i} \mid \mathbf{Q}_{i}\right)\right),
$$

where $\mathbf{Q}_{1}, \ldots, \mathbf{Q}_{k}$ are subsets of vector spaces, $\mathbf{Q}:=\mathbf{Q}_{1} \times \cdots \times \mathbf{Q}_{k}, x=\left(x_{1}, \ldots, x_{k}\right) \in$ $\mathbf{Q}, d=\left(d_{1}, \ldots, d_{k}\right) \in C(x \mid \mathbf{Q})$, and $x^{*}=\left(x_{1}^{*}, \ldots, x_{k}^{*}\right)$.

The paper is divided as follows. In section 2, auxiliary results needed for the main result are presented. In particular, when $\mathbf{Q}$ is given by (1.4) or (1.5), we recall the characterizations of both normal and critical cones $(N(x \mid \mathbf{Q})$ and $C(x \mid \mathbf{Q}))$, and the evaluation of the support function of $V(x, d \mid \mathbf{Q})$ in terms of the images of the setvalued map $Q$. Also, we state a special version of Theorem 1.1 which is tailored for the abstract control setting and which will be used later in proving the main result. However, when $\mathbf{Q}$ is given by (1.5), the multiplier $x^{*}$ in Theorem 1.1 corresponding to the set inclusion constraint is in general in $\left(\mathcal{L}^{\infty}\left(\Omega, \mathbb{R}^{\gamma}\right)\right)^{*}$. Therefore, it is important in this case to obtain a reasonable sufficiency criterion for $x^{*}$ to be represented by an integrable function. This is accomplished in section 3 by using a uniform solvability criterion. In section 4, the results of the preceding sections are used to obtain secondorder necessary conditions for optimality in a general optimal control problem with control and state set-valued constraints. These conditions are phrased in terms of the critical tangent cone. A specialization of Theorem 4.1 to the case of inequality constraints is presented in Corollary 4.1. Therein, only the extra term corresponding to the pure-state constraints remains present. This term is phrased in terms of the function $\sigma$ defined in (2.14). Finally, a numerical example is provided at the end of section 4 in order to illustrate the utility of these results.

2. Auxiliary results. When dealing with control problems, there are two special cases for $X$ and $\mathbf{Q}$ where the characterization of the critical cone $C(x \mid \mathbf{Q})$ and the evaluation of the support function of $V(x, d \mid \mathbf{Q})$ are imperative.

The first setting considers $X=\mathcal{C}\left(T, \mathbb{R}^{\kappa}\right)$, where $T=(T, \rho)$ is a compact metric space, and $Q: T \rightarrow 2^{\mathbb{R}^{\kappa}}$ is a lower semicontinuous set-valued function whose images are closed and convex with nonempty interior. Define the $\mathbf{Q} \subset \mathcal{C}\left(T, \mathbb{R}^{\kappa}\right)$ as the set of continuous selections of $Q$ by

$$
\mathbf{Q}=\operatorname{sel}_{C}(Q):=\left\{x \in \mathcal{C}\left(T, \mathbb{R}^{\kappa}\right) \mid x(t) \in Q(t) \text { for } t \in T\right\} .
$$

Then $\operatorname{sel}_{C}(Q)$ is a closed convex set of $\mathcal{C}\left(T, \mathbb{R}^{\kappa}\right)$.

Regarding $\mathbf{Q}=\operatorname{sel}_{C}(Q)$, a thorough study of convex analysis concepts (normal and tangent cones, support function, etc.) was developed in [PZ99a]. For instance, if 
we denote by $\frac{d \mu}{d|\mu|}$ the Radon-Nikodým derivative of $\mu$ with respect to $|\mu|$, it is shown that

$$
\mu \in N\left(x \mid \operatorname{sel}_{C}(Q)\right) \quad \text { if and only if } \quad \frac{d \mu}{d|\mu|}(t) \in N(x(t) \mid Q(t)) \quad \text { for } \mu \text {-a.e. } t \in T \text {. }
$$

Results concerning the second-order admissible variations, critical cone, and application to abstract optimization were derived in [PZ98]. The nonemptiness of the interior of the images of the set-valued function $Q$ implies, by [PZ99a, Theorem 4.2], that $\operatorname{sel}_{C}(Q)$ has a nonempty interior, too. A characterization of the set of critical directions is offered by the following results from [PZ98, Theorem 3.5, Lemmas 3.6 and 3.8]. Note that condition (2.3) below needs to be verified for $\xi \in \mathbb{R}^{\kappa}$, i.e., over a finite-dimensional space.

TheOrem 2.1. Let $x \in \operatorname{sel}_{C}(Q)$. Then $d \in \mathcal{C}\left(T, \mathbb{R}^{\kappa}\right)$ is in the critical cone $C\left(x \mid \operatorname{sel}_{C}(Q)\right)$ if and only if there exists a constant $M>0$ such that, for all $t \in T$,

$$
\langle\xi, d(t)\rangle^{2} \leq M|\xi|\left(\delta^{*}(\xi \mid Q(t))-\langle\xi, x(t)\rangle\right) \quad \text { whenever } \quad \xi \in \mathbb{R}^{\kappa} \text { and }\langle\xi, d(t)\rangle>0 .
$$

A consequence of Theorem 2.1 concerns the connection between $C\left(x \mid \operatorname{sel}_{C}(Q)\right)$ and the set-valued mapping $t \mapsto C(x(t) \mid Q(t))$. From Theorem 2.1 applied to $Q:=Q(t)$, $T=\{t\}$, and $d=d(t)$ (where $t$ is kept fixed), it results that $d(t) \in C(x(t) \mid Q(t))$ is equivalent to the fact that $(2.3)$ holds for some constant $M_{t}>0$. Therefore, Theorem 2.1 can be reformulated as follows:

$A$ continuous function d belongs to $C\left(x \mid \operatorname{sel}_{C}(Q)\right)$ if and only if

$$
d(t) \in C(x(t) \mid Q(t)) \quad(t \in T),
$$

and the corresponding constants $M_{t}$ from (2.3) can be chosen to be uniformly bounded.

When (2.3) is valid for some constant $M$ and for all $t \in T$, then we say that (2.4) holds uniformly in $t \in T$.

The second special setting is when $X=\mathcal{L}^{\infty}\left(\Omega, \mathbb{R}^{\gamma}\right)$, where $(\Omega, \mathcal{A}, \nu)$ is a complete finite measure space, and $Q: \Omega \rightarrow 2^{\mathbb{R}^{\gamma}}$ is a measurable set-valued function whose images are closed sets with nonempty interior and $\operatorname{sel}_{\infty}(Q)$ is defined by

$$
\mathbf{Q}=\operatorname{sel}_{\infty}(Q):=\left\{x \in \mathcal{L}^{\infty}\left(\Omega, \mathbb{R}^{\gamma}\right) \mid x(t) \in Q(t) \text { for a.e. } t \in \Omega\right\} .
$$

For this case, the concept of convex analysis was studied in [PZ99b], [PZ99c]. In particular, for $x \in \operatorname{sel}_{\infty}(Q)$ and for $\varphi \in L^{1}\left(\Omega, \mathbb{R}^{\gamma}\right)$,

$$
\varphi \in N\left(x \mid \operatorname{sel}_{\infty}(Q)\right) \quad \text { if and only if } \quad \varphi(t) \in N(x(t) \mid Q(t)) \quad \text { for a.e. } t \in \Omega .
$$

For the second-order admissible variations, critical cone, and the application to secondorder optimality conditions in an abstract setting, results were obtained in [PZ00].

In order that the interior of $\operatorname{sel}_{\infty}(Q)$ be nonempty it is necessary and sufficient (by [PZ99c, Theorem 3]; see also [PZ99b]) to assume that $Q$ satisfies

$$
\exists r \geq \rho>0 \text { and, for a.e. } t \in \Omega, \exists x_{t} \in \mathbb{R}^{\gamma} \text { such that } B_{\rho}\left(x_{t}\right) \subset Q(t) \cap B_{r},
$$


where $B_{\rho}(x)$ stands for the ball centered at $x$ of radius $\rho$, and $B_{r}$ stands for the ball centered at 0 of radius $r$.

The following consists of a characterization of $C\left(x \mid \operatorname{sel}_{\infty}(Q)\right)$. It provides a verifiable condition over a finite-dimensional space. As was the case in Theorem 2.1 for the space of continuous functions, (2.7) below is to be checked for elements $\xi \in \mathbb{R}^{\gamma}$ even though the underlying space is $\mathcal{L}^{\infty}\left(\Omega, \mathbb{R}^{\gamma}\right)$.

TheOREM 2.2. Let $Q: \Omega \rightarrow 2^{\mathbb{R}^{\gamma}}$ be a measurable set-valued map whose images are closed convex sets and satisfy (2.6). Let $x \in \operatorname{sel}_{\infty}(Q)$ and $d \in \mathcal{L}^{\infty}\left(\Omega, \mathbb{R}^{\gamma}\right)$. Then $d \in C\left(x \mid \operatorname{sel}_{\infty}(Q)\right)$ if and only if there exists a constant $M>0$ such that, for a.e. $t \in \Omega$, the following condition is valid:

$$
\langle\xi, d(t)\rangle^{2} \leq M|\xi|\left(\delta^{*}(\xi \mid Q(t))-\langle\xi, x(t)\rangle\right) \quad \text { whenever } \xi \in \mathbb{R}^{\gamma} \text { and }\langle\xi, d(t)\rangle>0 .
$$

From Theorem 2.2 it readily follows that, for a.e. $t \in \Omega, d(t) \in C(x(t) \mid Q(t))$ if and only if (2.7) holds for some $M_{t}>0$ on the domain indicated. Therefore, Theorem 2.2 can be rephrased as

$A$ bounded measurable function d belongs to $C\left(x \mid \operatorname{sel}_{\infty}(Q)\right)$ if and only if

$$
d(t) \in C(x(t) \mid Q(t)) \quad \text { for a.e. }(t \in \Omega),
$$

and the corresponding constants $M_{t}$ from (2.7) can be chosen to be uniformly bounded on a set of full measure.

When (2.7) is valid for some constant $M$ and for a.e. $t \in \Omega$, then we say that (2.8) holds almost uniformly on $\Omega$.

The rest of this section is devoted to recalling the results on the calculation of the support functional to the second-order admissible variation set of $\operatorname{sel}_{C}(Q)$ and $\operatorname{sel}_{\infty}(Q)$, respectively.

We introduce the following notation. Let $Q$ be a subset of $\mathbb{R}^{\gamma}, x \in Q$, and $d \in \mathbb{R}^{\gamma}$. Denote

$$
E(x, d \mid Q)(\xi):=\frac{\langle\xi, d\rangle^{2}}{4\left[\langle\xi, x\rangle-\delta^{*}(\xi \mid Q)\right]} \quad \text { for } \quad \xi \in \mathbb{R}^{\gamma} \text { such that } \xi \notin N(x \mid Q) .
$$

Note that $E(x, d \mid Q)(\xi)$ is well defined, because $\langle\xi, x\rangle-\delta^{*}(\xi \mid Q) \neq 0$ if and only if $\xi \notin N(x \mid Q)$. If $d \in T(x \mid Q)$ and $\langle\xi, d\rangle>0$, then $\xi \notin N(x \mid Q)$; hence, in this case, $E(x, d \mid Q)(\xi)$ is defined for $\langle\xi, d\rangle>0$.

Set

$$
d^{\perp}:=\left\{\xi \in \mathbb{R}^{\gamma} \mid\langle\xi, d\rangle=0\right\}, \quad d^{>}:=\left\{\xi \in \mathbb{R}^{\gamma} \mid\langle\xi, d\rangle>0\right\},
$$

and define from $\mathbb{R}^{\gamma}$ to the extended reals the function

$$
\mathcal{E}(x, d \mid Q)(\xi):=\left\{\begin{array}{cl}
\liminf _{\substack{\zeta \rightarrow \xi \\
\zeta \in d^{>}}} E(x, d \mid Q)(\zeta) & \text { if } \xi \in N(x \mid Q) \cap d^{\perp}, \\
+\infty, & \text { otherwise. }
\end{array}\right.
$$

One can see that $\mathcal{E}(x, d \mid Q)(\cdot)$ is a positively homogeneous and also lower semicontinuous function on $\mathbb{R}^{\gamma} \backslash\{0\}$.

Define the convex regularization $\overline{\mathrm{co}} \mathcal{E}(x, d \mid Q)(\cdot)$ to be the largest lower semicontinuous convex function below $\mathcal{E}(x, d \mid Q)(\cdot)$; that is,

$$
\begin{aligned}
\overline{\operatorname{co}} \mathcal{E}(x, d \mid Q)(\xi)=\sup \left\{\varphi(\xi) \mid \varphi: \mathbb{R}^{\gamma}\right. & \rightarrow[-\infty, \infty] \text { is convex and lsc, } \\
\varphi(\zeta) & \left.\leq \mathcal{E}(x, d \mid Q)(\zeta) \forall \zeta \in \mathbb{R}^{\gamma} \backslash\{0\}\right\} .
\end{aligned}
$$

It results that $\overline{\mathrm{co}} \boldsymbol{E}(x, d \mid Q)(\cdot)$ is also sublinear. 
The following result offers an evaluation of the support function of the set $V\left(x, d \mid \operatorname{sel}_{\infty}(Q)\right)$ at linear functionals that can be represented in terms of integrable functions (cf. [PZ00, Corollary 2.7]).

THEOREM 2.3. Let $Q$ be a closed convex set-valued measurable set-valued map on $\Omega, x \in \operatorname{sel}_{\infty}(Q)$, and $d \in C\left(x \mid \operatorname{sel}_{\infty}(Q)\right)$, and let $\varphi \in \mathcal{L}^{1}\left(\Omega, \mathbb{R}^{\gamma}\right)$. Then

$$
\delta^{*}\left(\varphi \mid V\left(x, d \mid \operatorname{sel}_{\infty}(Q)\right)\right)=\int_{\Omega} \overline{\operatorname{co}} \mathcal{E}(x(t), d(t) \mid Q(t))(\varphi(t)) d \nu(t) .
$$

A common type of constraint is when $Q$ comes from inequality constraints, that is, when $Q(t)=\mathbb{R}_{-}^{\gamma}$ for all $t \in \Omega$. In this case the description of the critical cone and the evaluation of the support function simplify drastically.

COROLlary 2.1. Let $x \in \operatorname{sel}_{\infty}\left(\mathbb{R}_{-}^{\gamma}\right)$. Then a bounded measurable function $d=$ $\left(d_{1}, \ldots, d_{\gamma}\right): \Omega \rightarrow \mathbb{R}^{\gamma}$ is in $C\left(x \mid \operatorname{sel}_{\infty}\left(\mathbb{R}_{-}^{\gamma}\right)\right)$ if and only if

(i) there exists a constant $M \geq 0$ such that, for all $i=1, \ldots, \gamma$ and for a.e. $t \in \Omega, d_{i}^{2}(t) \leq-M x_{i}(t)$ whenever $x_{i}(t) \leq 0$ and $d_{i}(t)>0$ hold;

(ii) for a.e. $t \in \Omega$ with $x_{i}(t)=0$, we have $d_{i}(t) \leq 0$.

Furthermore, let $x \in \operatorname{sel}_{\infty}\left(\mathbb{R}_{-}^{\gamma}\right), d \in C\left(x \mid \operatorname{sel}_{\infty}\left(\mathbb{R}_{-}^{\gamma}\right)\right)$, and let $\varphi: \Omega \rightarrow \mathbb{R}_{+}^{\gamma}$ be an integrable function such that $\varphi^{T}(t) x(t)=0$ and $\varphi^{T}(t) d(t)=0$ for a.e. $t \in \Omega$. Then

$$
\delta^{*}\left(\varphi \mid V\left(x, d \mid \operatorname{sel}_{\infty}\left(\mathbb{R}_{-}^{\gamma}\right)\right)\right)=0 .
$$

Proof. Using the product rule for the critical cone and the first part of [PZ94b, Lemma 7], we get that the inclusion $d \in C\left(x \mid \operatorname{sel}_{\infty}\left(\mathbb{R}_{-}^{\gamma}\right)\right)$ is characterized by conditions (i) and (ii).

Observe that the nonnegativity of $\varphi$ and the conditions $\varphi^{T} x=0$ and $\varphi^{T} d=0$ yield that $\varphi_{i}^{T} x_{i}=0$ and $\varphi_{i}^{T} d_{i}=0$ for all $i=1, \ldots, \gamma$ almost everywhere in $\Omega$. Thus, applying the sum rule for the evaluation of the support function of secondorder variation sets and the second part of [PZ94b, Lemma 7], the second statement of the corollary will follow.

The analogous result for the case of $\mathbf{Q}=\operatorname{sel}_{C}(Q)$ requires more involved notions (see [PZ98]). Let $T$ be a compact metric space, and let $Q: T \rightarrow 2^{\mathbb{R}^{\kappa}}$ be a setvalued function whose images are closed and convex sets with nonempty interior. Let $x \in \operatorname{sel}_{C}(Q)$ and $d \in C\left(x \mid \operatorname{sel}_{C}(Q)\right)$. Denote by $d^{\#}: T \rightarrow 2^{\mathbb{R}^{\kappa}}$ the following set-valued function:

$$
d^{\#}(t)=\left\{\xi \in \mathbb{R}^{\kappa} \mid \exists t_{n} \rightarrow t, \exists \xi_{n} \rightarrow \xi \text { with } \xi_{n} \in d\left(t_{n}\right)^{>} \forall n\right\} .
$$

Define

$$
\mathbb{E}(x, d \mid Q)(t, \xi):=\left\{\begin{array}{cc}
\liminf _{\substack{s, \zeta) \rightarrow(t, \xi) \\
\zeta \in d(s))^{>}}} E(x(s), d(s) \mid Q(s))(\zeta) \text { if } \xi \in N(x(t) \mid Q(t)) \cap d(t)^{\perp} \cap d^{\#}(t), \\
0 & \text { if } \xi \in N(x(t) \mid Q(t)) \cap d(t)^{\perp} \backslash d^{\#}(t), \\
+\infty, & \text { otherwise. }
\end{array}\right.
$$

Define the convex regularization $\overline{\mathrm{co}} \mathbb{E}(x, d \mid Q)(\cdot, \cdot)$ to be the largest lower semicontinuous function $\varphi: T \times \mathbb{R}^{\kappa} \rightarrow[-\infty, \infty]$ below $\mathbb{E}(x, d \mid Q)(\cdot, \cdot)$ such that, for each $t \in T$, the function $\xi \mapsto \varphi(t, \xi)$ is convex on $\mathbb{R}^{\kappa}$.

In the following result (cf. [PZ98, Theorem 3.10]), we describe how the support functional of $V\left(x, d \mid \operatorname{sel}_{C}(Q)\right)$ can be evaluated in terms of $\overline{\mathrm{co}} \mathbb{E}$. 
TheOREM 2.4. Let $T$ be a compact metric space, and let $Q: T \rightarrow 2^{R^{\kappa}}$ be a lower semicontinuous set-valued function whose images are closed and convex with nonempty interior. Let $x \in \operatorname{sel}_{C}(Q), d \in C\left(x \mid \operatorname{sel}_{C}(Q)\right)$, and let $\mu$ be a bounded vector-valued Borel measure on T. Then

$$
\delta^{*}\left(\mu \mid V\left(x, d \mid \operatorname{sel}_{C}(Q)\right)\right)=\int_{T} \overline{\operatorname{co}} \mathbb{E}(x, d \mid Q)\left(t, \frac{d \mu}{d|\mu|}(t)\right) d|\mu|(t),
$$

where $\frac{d \mu}{d|\mu|}(\cdot)$ is the Radon-Nikodým derivative of $\mu$ with respect to its total variation $|\mu|$.

For given continuous functions $a, b: T \rightarrow \mathbb{R}$, define $\sigma_{a, b}: T \rightarrow[-\infty, \infty]$ by

$$
\sigma_{a, b}(t):=\left\{\begin{array}{cl}
\liminf _{\tau, \vec{t}} \frac{b^{2}(\tau)}{4 a(\tau)} & \text { if } t \in T_{a=0, b=0} \cap \partial\left(T_{a<0, b>0}\right), \\
0 & \text { if } t \in T_{a=0, b=0} \backslash \partial\left(T_{a<0, b>0}\right), \\
+\infty, & \text { otherwise, }
\end{array}\right.
$$

where

$$
T_{a=0, b=0}:=\{t \in T \mid a(t)=0, b(t)=0\}, \quad T_{a<0, b>0}:=\{t \in T \mid a(t)<0, b(t)>0\} .
$$

Corollary 2.2. Let $x=\left(x_{1}, \ldots, x_{\kappa}\right) \in \operatorname{sel}_{C}\left(\mathbb{R}_{-}^{\kappa}\right)$. Then a continuous function $d=\left(d_{1}, \ldots, d_{\kappa}\right): T \rightarrow \mathbb{R}^{\kappa}$ is in $C\left(x \mid \operatorname{sel}_{C}\left(\mathbb{R}_{-}^{\kappa}\right)\right)$ if and only if

(i) there exists a constant $M \geq 0$ such that, for all $i=1, \ldots, \kappa$ and for all $t \in T$, $d_{i}^{2}(t) \leq-M x_{i}(t)$ whenever $x_{i}(t) \leq 0$ and $d_{i}(t)>0$ hold;

(ii) for all $t \in T$ with $x_{i}(t)=0$, we have $d_{i}(t) \leq 0$.

Furthermore, let $x \in \operatorname{sel}_{C}\left(\mathbb{R}_{-}^{\kappa}\right), d \in C\left(x \mid \operatorname{sel}_{C}\left(\mathbb{R}_{-}^{\kappa}\right)\right)$, and let $\mu=\left(\mu_{1}, \ldots, \mu_{\kappa}\right)$ be a bounded vector-valued Borel measure on $T$ with nonnegative components such that $\operatorname{supp} \mu_{i} \subset\left\{t \in T \mid x_{i}(t)=0, d_{i}(t)=0\right\}$ for all $i=1, \ldots, \kappa$. Then

$$
\delta^{*}\left(\mu \mid V\left(x, d \mid \operatorname{sel}_{C}\left(\mathbb{R}_{-}^{\kappa}\right)\right)\right)=\sum_{i=1}^{\kappa} \int_{T} \sigma_{x_{i}, d_{i}}(t) d \mu_{i}(t) .
$$

Proof. Using the product rule for the critical cone and [PZ98, Corollary 4.2(i)], we get that the inclusion $d \in C\left(x \mid \operatorname{sel}_{C}\left(\mathbb{R}_{-}^{\kappa}\right)\right)$ is characterized by conditions (i) and (ii).

Applying the sum rule for the evaluation of the support function of second-order variation sets and [PZ98, Corollary 4.2(iv)], the second statement of the corollary will also follow.

In the rest of this section we present second-order optimality conditions for the following abstract control problems, which are a special form of the problem $(\mathcal{P})$. This problem allows the distinction between the control and the state variables:

Assume that $X, U, Y, V$, and $W$ are Banach spaces (over $\mathbb{R}$ ), and $D \subset X \times U$ is nonempty and open. Let $\mathbf{F}: D \rightarrow \mathbb{R}, \mathbf{G}: D \rightarrow V, \mathbf{H}: D \rightarrow W, \mathbf{K}: D \rightarrow Y$, and, further, that $\mathbf{Q} \subset V$ is a closed convex set with nonempty interior. The problem $(\mathcal{P})$ is to minimize $F(x, u)$ in $(x, u) \in D$ subject to

(i) $\mathbf{G}(x, u) \in \mathbf{Q} \quad$ (Banach space-valued mixed state-control inequality and control set constraint),

(ii) $\mathbf{H}(x, u)=0 \quad$ (Banach space-valued mixed state-control equality),

(iii) $\mathbf{K}(x, u)=0 \quad$ (control system). 
The admissibility and optimality of a pair $(x, u) \in D$ is defined similarly to that of problem $(\mathcal{P})$.

The second constraint $\mathbf{G}(x, u) \in \mathbf{Q}$ is able to handle Banach space-valued inequalities and control set constraint as well. For instance, if $\mathbf{Q}$ is a closed convex cone with nonempty interior, then introducing the ordering $\leq_{\mathbf{Q}}$ in $V$ by $x \leq_{\mathbf{Q}} y \Longleftrightarrow x-y \in \mathbf{Q}$, one can see that our first constraint can be rewritten as $\mathbf{G}(x, u) \leq_{\mathbf{Q}} 0$. On the other hand the constraints $u \in Q$ or $x \in Q$, where $Q$ is a convex set with nonempty interior, are obviously a particular case of (i). In this case problem $(\mathcal{P})$ specializes to the mixed problem dealt with in [IT79, section 1.1.3, p. 70]. However, both the regularity assumptions and the results are of a different nature from those in our case.

At this stage one cannot make any difference between the mixed state-control equality and control system constraints. However, the difference becomes clear when evoking the regularity conditions stated below.

A pair $(\widehat{x}, \widehat{u}) \in D$ is called regular for problem $(\mathcal{P})$ if the following conditions are satisfied:

$\left(\mathbf{R}_{1}\right) \mathbf{G}$ is strictly Fréchet differentiable at $(\widehat{x}, \widehat{u})$;

$\left(\mathbf{R}_{2}\right) \mathbf{H}$ is strictly Fréchet differentiable at $(\widehat{x}, \widehat{u})$ and the partial Fréchet derivative $\mathbf{H}_{u}(\widehat{x}, \widehat{u}): U \rightarrow W$ has the full rank property; that is, it has a bounded right inverse;

$\left(\mathbf{R}_{3}\right) \mathbf{K}$ is strictly Fréchet differentiable at $(\widehat{x}, \widehat{u})$ and the equation is an abstract control system at $(\widehat{x}, \widehat{u})$; i.e., the partial derivative $\mathbf{K}_{x}(\widehat{x}, \widehat{u})$ is a Fredholm operator and $\mathbf{K}_{u}(\widehat{x}, \widehat{u})$ is compact.

We note that when $\mathbf{K}$ fulfills the above assumption at each point of $D$, then the equation $\mathbf{K}=0$ will be called a (global) control system. It is worth noting that if $\mathbf{K}$ is continuously Fréchet differentiable on $D, \mathbf{K}_{x}$ is a Fredholm operator, and $D$ is a connected set, then ind $\mathbf{K}_{x}$ is constant on $D$ and hence the index of a control system could be defined.

We indicate by $\widehat{\Phi}$ the evaluation of the function $\Phi$ at $(\widehat{x}, \widehat{u})$.

Let $(\widehat{x}, \widehat{u})$ be a regular admissible pair for problem $(\mathcal{P})$. A direction $(\delta x, \delta u) \in$ $X \times U$ is called regular for our problem $(\mathcal{P})$ at $(\widehat{x}, \widehat{u})$ if

$\left(\mathbf{R}_{4}\right)$ the second-order directional derivatives $\widehat{\mathbf{G}}^{\prime \prime}(\delta x, \delta u), \widehat{\mathbf{H}}^{\prime \prime}(\delta x, \delta u)$, and $\widehat{\mathbf{K}}^{\prime \prime}(\delta x, \delta u)$ of $\mathbf{G}, \mathbf{H}$, and $\mathbf{K}$, respectively, exist at $(\widehat{x}, \widehat{u})$ in the direction $(\delta x, \delta u)$.

A direction $(\delta x, \delta u) \in X \times U$ is called critical for $(\mathcal{P})$ at $(\widehat{x}, \widehat{u})$ if

$\left(\mathbf{C}_{1}\right) \widehat{\mathbf{F}}_{x} \delta x+\widehat{\mathbf{F}}_{u} \delta u \leq 0$;

$\left(\mathbf{C}_{2}\right) \widehat{\mathbf{G}}_{x} \delta x+\widehat{\mathbf{G}}_{u} \delta u \in C(\widehat{\mathbf{G}} \mid \mathbf{Q}), \widehat{\mathbf{H}}_{x} \delta x+\widehat{\mathbf{H}}_{u} \delta u=0, \widehat{\mathbf{K}}_{x} \delta x+\widehat{\mathbf{K}}_{u} \delta u=0$.

One can check that $(\delta x, \delta u)=(0,0)$ is always a regular and critical direction at $(\widehat{x}, \widehat{u})$ for $(\mathcal{P})$.

The next result is the multiplier rule for problem $(\mathcal{P})$ obtained in [PZ94b, Theorem $3]$.

THEOREM 2.5. Let $(\widehat{x}, \widehat{u})$ be a regular solution for problem $(\mathcal{P})$. Then, for every regular critical direction $(\delta x, \delta u) \in X \times U$, there exist Lagrange multipliers $v^{*} \in V^{*}$, $w^{*} \in W^{*}$, and $y^{*} \in Y^{*}$ such that at least one of them is different from zero and the following relations hold:

$$
\begin{gathered}
\left\langle v^{*}, v\right\rangle \leq 0 \quad \text { for } \quad v \in \mathbf{Q}-\widehat{\mathbf{G}}, \quad\left\langle v^{*}, \widehat{\mathbf{G}}_{x} \delta x+\widehat{\mathbf{G}}_{u} \delta u\right\rangle=0, \\
\lambda \widehat{\mathbf{F}}_{x}+v^{*} \circ \widehat{\mathbf{G}}_{x}+w^{*} \circ \widehat{\mathbf{H}}_{x}+y^{*} \circ \widehat{\mathbf{K}}_{x}=0, \\
\lambda \widehat{\mathbf{F}}_{u}+v^{*} \circ \widehat{\mathbf{G}}_{u}+w^{*} \circ \widehat{\mathbf{H}}_{u}+y^{*} \circ \widehat{\mathbf{K}}_{u}=0,
\end{gathered}
$$


and

$$
\begin{aligned}
\lambda \widehat{\mathbf{F}}^{\prime \prime}(\delta x, \delta u)+\left\langle v^{*}, \widehat{\mathbf{G}}^{\prime \prime}(\delta x, \delta u)\right\rangle+\left\langle w^{*}, \widehat{\mathbf{H}}^{\prime \prime}(\delta x, \delta u)\right\rangle+\left\langle y^{*}, \widehat{\mathbf{K}}^{\prime \prime}(\delta x, \delta u)\right\rangle \\
\geq 2 \delta^{*}\left(v^{*} \mid V\left(\widehat{\mathbf{G}}, \widehat{\mathbf{G}}_{x} \delta x+\widehat{\mathbf{G}}_{u} \delta u \mid \mathbf{Q}\right)\right) .
\end{aligned}
$$

3. Uniform solvability criteria. In the next result, we characterize the solvability of a system of linear equations over cones in different ways.

Theorem 3.1. Let $G \in \mathbb{R}^{\gamma \times m}, H \in \mathbb{R}^{\delta \times m}$, and $D \in \mathbb{R}^{\gamma \times q}$ be matrices and let $C \subset \mathbb{R}^{q}$ be a closed convex cone. Then the following four statements are equivalent to each other:

(i) For all vectors $v \in \mathbb{R}^{\gamma}$ and $w \in \mathbb{R}^{\delta}$, there exist $a \in \mathbb{R}^{m}$ and $c \in C$ such that

$$
v=G a-D c \quad \text { and } \quad w=H a .
$$

(ii) If $\xi \in \mathbb{R}^{\gamma}, \eta \in \mathbb{R}^{\delta}$, then

$$
\xi^{T} G+\eta^{T} H=0 \quad \text { and } \quad \xi^{T} D \in C^{\circ}
$$

are valid if and only if $(\xi, \eta)=(0,0)$. (Here $C^{\circ}$ denotes the polar cone of C.)

(iii) There exists a constant $\tau>0$ such that

$$
\left|\xi^{T} G+\eta^{T} H\right|^{2}+\left[\operatorname{dist}\left(\xi^{T} D, C^{\circ}\right)\right]^{2} \geq \tau|(\xi, \eta)|^{2} \quad\left((\xi, \eta) \in \mathbb{R}^{\gamma} \times \mathbb{R}^{\delta}\right) .
$$

(iv) The matrix $H$ is of full rank and there exist two maps a: $\mathbb{R}^{\gamma} \times \mathbb{R}^{\delta} \rightarrow \mathbb{R}^{m}$, $c: \mathbb{R}^{\gamma} \times \mathbb{R}^{\delta} \rightarrow \mathbb{R}^{q}$ and a constant $\rho>0$ such that

$$
\begin{aligned}
& G a(v, w)-D c(v, w)=v, \quad H a(v, w)=w, \quad c(v, w) \in C \quad\left((v, w) \in \mathbb{R}^{\gamma} \times \mathbb{R}^{\delta}\right), \\
& \text { and }\left\|\left(H H^{T}\right)^{-1}\right\| \leq \rho, \\
& \qquad \begin{aligned}
|a(v, w)| & \leq \rho[\|G\|+\|H\|]|(v, w)|, \\
|c(v, w)| & \leq \rho\|D\||(v, w)| \quad\left((v, w) \in \mathbb{R}^{\gamma} \times \mathbb{R}^{\delta}\right) .
\end{aligned}
\end{aligned}
$$

Moreover, if (iii) holds, then $\rho$ can be chosen such that $\rho \leq 1 / \tau$.

Remark 3.1. As we shall soon see in the proof below, the equivalence (i) $\Longleftrightarrow$ (ii) $\Longleftrightarrow$ (iii) and the implication (iv) $\Longrightarrow$ (i) are straightforward. Note that the equivalence between (i) and (iv) could be obtained via an open-mapping theorem for convex processes (i.e., the Robinson-Ursescu theorem). However, the main contribution of Theorem 3.1 lies in the implication (iii) $\Longrightarrow$ (iv), and more specifically in the fact that the constant $\rho$ turns out to be less than or equal to $1 / \tau$, where $\tau$ is the constant in (3.3). This fact becomes crucial when applying the result of Theorem 3.1 to data that consist of essentially bounded matrix-valued functions.

Proof. (i) $\Longleftrightarrow$ (ii). Assume that (i) is true and let $\xi \in \mathbb{R}^{\gamma}$ and $\eta \in \mathbb{R}^{\delta}$ such that (3.2) holds. Let $v \in \mathbb{R}^{\gamma}$ and $w \in \mathbb{R}^{\delta}$ be arbitrary. By (i), there exist $a \in \mathbb{R}^{m}$ and $c \in C$ such that (3.1) holds. Multiplying these equations by $\xi$ and $\eta$, respectively, we get

$$
\xi^{T} v+\eta^{T} w=\xi^{T} G a-\xi^{T} D c+\eta^{T} H a=-\xi^{T} D c \geq 0 .
$$

Hence $\xi^{T} v+\eta^{T} w \geq 0$ for all $v$ and $w$. This implies that $\xi=0$ and $\eta=0$. 
Conversely, assume that (ii) holds but (i) is not true. Then the set

$$
K:=\left\{(G a-D c, H a) \mid a \in \mathbb{R}^{m}, c \in C\right\}
$$

is a proper subcone of $\mathbb{R}^{\gamma} \times \mathbb{R}^{\delta}$. Thus, there exists $(\xi, \eta) \neq(0,0)$ such that $(\xi, \eta) \in$ $-K^{\circ}$, that is,

$$
\xi^{T}(G a-D c)+\eta^{T} H a \geq 0
$$

for all $a \in \mathbb{R}^{m}, c \in C$. This yields the fact that (3.2) is valid. Hence, by (ii), $\xi=0$ and $\eta=0$. The contradiction shows that (ii) implies (i).

(ii) $\Longleftrightarrow$ (iii). If (ii) holds, then

$$
\varphi(\xi, \eta):=\left|\xi^{T} G+\eta^{T} H\right|^{2}+\left[\operatorname{dist}\left(\xi^{T} D, C^{\circ}\right)\right]^{2}>0
$$

for all $(\xi, \eta) \neq(0,0)$. Hence, the infimum of $\varphi$ on the unit sphere of $\mathbb{R}^{\gamma} \times \mathbb{R}^{\delta}$, which we denote by $\tau$, is positive. Using quadratic homogeneity, the statement of (iii) follows. The reverse implication holds trivially.

Thus, we have obtained that conditions (i), (ii), and (iii) are equivalent.

(iii) $\Longrightarrow$ (iv). Putting $\xi=0$ into (3.3), we get

$$
\left|\eta^{T} H\right|^{2} \geq \tau|\eta|^{2}, \quad \text { i.e., } \quad \eta^{T} H H^{T} \eta \geq \tau|\eta|^{2} \quad\left(\eta \in \mathbb{R}^{\delta}\right) .
$$

Hence, $H H^{T}$ is positive definite, invertible, and $\left\|\left(H H^{T}\right)^{-1}\right\| \leq \rho$, where $\rho:=1 / \tau$.

Let $v \in \mathbb{R}^{\gamma}$ and $w \in \mathbb{R}^{\delta}$ be fixed arbitrarily. Using the equivalence of (i) and (iii), we can see that there exist $x \in \mathbb{R}^{m}$ and $y \in C$ such that $G x-D y=v$ and $H x=w$. Thus, the following optimization problem has a unique solution $(x, y)$ :

$$
\frac{1}{2}\left(\|x\|^{2}+\|y\|^{2}\right) \longrightarrow \min \quad \text { w.r.t. } \quad G x-D y=v, \quad H x=w, \quad y \in C .
$$

(The uniqueness follows from the strict convexity of the objective function.) Hence, there are multipliers $\lambda \geq 0, \xi \in \mathbb{R}^{\gamma}, \eta \in \mathbb{R}^{\delta}$, and $\zeta \in C^{\circ}$, not all zero, such that

$$
\lambda x^{T}+\xi^{T} G+\eta^{T} H=0, \quad \lambda y^{T}-\xi^{T} D+\zeta^{T}=0, \quad \zeta^{T} y=0 .
$$

If $\lambda$ were zero, then $\xi^{T} G+\eta^{T} H=0$ and $\xi^{T} D \in C^{\circ}$, which, due to (3.3), yields $\xi=0$, $\eta=0$. Thus also $\zeta=0$, which is a contradiction. Thus, we may assume that $\lambda=1$. Then

$$
\begin{aligned}
-(\xi, \eta)^{T}(v, w) & =-\xi^{T}(G x-D y)-\eta^{T} H x=-\left(\xi^{T} G+\eta^{T} H\right) x+\left(y^{T}+\zeta^{T}\right) y \\
& =\left|\xi^{T} G+\eta^{T} H\right|^{2}+|y|^{2}=\left|\xi^{T} G+\eta^{T} H\right|^{2}+\left|D^{T} \xi-\zeta\right|^{2} \\
& \geq\left|\xi^{T} G+\eta^{T} H\right|^{2}+\left[\operatorname{dist}\left(\xi^{T} D, C^{\circ}\right)\right]^{2} \geq \tau|(\xi, \eta)|^{2} .
\end{aligned}
$$

Using the Cauchy-Schwarz inequality, this yields

$$
|(\xi, \eta)||(v, w)| \geq \tau|(\xi, \eta)|^{2}, \quad \text { i.e., } \quad|(\xi, \eta)| \leq \rho|(v, w)| .
$$

Hence

$$
|x|=\left|G^{T} \xi+H^{T} \eta\right| \leq \rho[\|G\|+\|H\|]|(v, w)|
$$

and

$|y|^{2}=y^{T}\left(D^{T} \xi-\zeta\right)=\left|y^{T} D^{T} \xi\right| \leq|y|\|D\||\xi| \quad \Longrightarrow \quad|y| \leq\|D\||\xi| \leq \rho\|D\||(v, w)|$. 
Define $a(v, w)$ and $c(v, w)$ (for fixed $v \in \mathbb{R}^{\gamma}$ and $w \in \mathbb{R}^{\delta}$ ) to be, respectively, the solutions $x$ and $y$ of the optimization problem (3.6). Then the feasibility of $(x, y)$ yields (3.4); furthermore, the estimates (3.8) and (3.9) imply (3.5).

Finally, we note that the implication (iv) $\Longrightarrow$ (i) is obvious. Thus the proof of the theorem is complete.

Now we apply the implication (iii) $\Longrightarrow$ (iv) of the above result to essentially bounded matrix functions $G, H$, and $D$, where $C$ is the nonnegative orthant in $\mathbb{R}^{q}$. Below, $\mathcal{B}$ denotes the $\sigma$-algebra of Borel sets. The notation $x^{+}$stands for the nonnegative part of a real number $x$, that is, $x^{+}:=\max (0, x)$.

Theorem 3.2. Let $(\Omega, \mathcal{A}, \nu)$ be a finite measure space, $G: \Omega \rightarrow \mathbb{R}^{\gamma \times m}, H: \Omega \rightarrow$ $\mathbb{R}^{\delta \times m}$, and let $d_{1}, \ldots, d_{q}: \Omega \rightarrow \mathbb{R}^{\gamma}$ be bounded measurable functions. Assume that there exists a constant $\tau>0$ such that, for a.e. $t \in \Omega$,

$$
\left|\xi^{T} G(t)+\eta^{T} H(t)\right|^{2}+\sum_{i=1}^{q}\left[\left(\xi^{T} d_{i}(t)\right)^{+}\right]^{2} \geq \tau|(\xi, \eta)|^{2} \quad\left((\xi, \eta) \in \mathbb{R}^{\gamma} \times \mathbb{R}^{\delta}\right) .
$$

Then $H H^{T}: \Omega \rightarrow \mathbb{R}^{\delta \times \delta}$ has a bounded measurable inverse, and there exist $\mathcal{A} \times \mathcal{B} \times \mathcal{B}$ measurable maps a $: \Omega \times \mathbb{R}^{\gamma} \times \mathbb{R}^{\delta} \rightarrow \mathbb{R}^{m}$ and $c_{1}, \ldots, c_{q}: \Omega \times \mathbb{R}^{\gamma} \times \mathbb{R}^{\delta} \rightarrow[0, \infty)$ and a constant $R>0$ such that, for a.e. $t \in \Omega$,

$$
G(t) a(t, v, w)=v+\sum_{i=1}^{q} c_{i}(t, v, w) d_{i}(t), \quad H(t) a(t, v, w)=w \quad\left((v, w) \in \mathbb{R}^{\gamma} \times \mathbb{R}^{\delta}\right),
$$

and

$|a(t, v, w)| \leq R|(v, w)|, \quad c_{i}(t, v, w) \leq R|(v, w)| \quad\left((v, w) \in \mathbb{R}^{\gamma} \times \mathbb{R}^{\delta}, i=1, \ldots, q\right)$.

Proof. Set $\rho:=1 / \tau$,

$$
\begin{aligned}
C & :=\mathbb{R}_{+}^{q}=\left\{c=\left(c_{1}, \ldots, c_{q}\right) \mid c_{1}, \ldots, c_{q} \geq 0\right\}, \\
\text { and } \quad D(t) & :=\left(d_{1}(t), \ldots, d_{q}(t)\right) \quad(t \in \Omega) .
\end{aligned}
$$

Define the set-valued map $\Phi$ on $\Omega \times \mathbb{R}^{\gamma} \times \mathbb{R}^{\delta}$ by

$$
\begin{aligned}
\Phi(t, v, w):=\left\{(a, c) \in \mathbb{R}^{m} \times C:\right. & H(t) a=w, G(t) a-D(t) c=v, \\
& \left.|a| \leq \rho\left[\|G\|_{\infty}+\|H\|_{\infty}\right]|(v, w)|,|c| \leq \rho\|D\|_{\infty}|(v, w)|\right\} .
\end{aligned}
$$

We show that, for a.e. $t \in \Omega$, for all $v \in \mathbb{R}^{\gamma}$, and for all $w \in \mathbb{R}^{\delta}$, the set $\Phi(t, v, w)$ is nonempty.

Without loss of generality, we may assume that

$$
\|G(t)\| \leq\|G\|_{\infty}, \quad\|H(t)\| \leq\|H\|_{\infty}, \quad\|D(t)\| \leq\|D\|_{\infty},
$$

and (3.10) is valid for all $t \in \Omega$. (In fact, (3.10) is valid on a subset of $\Omega$, which is of full measure and which we do not relabel.)

Since $C^{\circ}=\left\{\left(c_{1}, \ldots, c_{q}\right) \mid c_{1}, \ldots, c_{q} \leq 0\right\}$, then

$$
\operatorname{dist}\left(x, C^{\circ}\right)=\sum_{i=1}^{q}\left(x_{i}^{+}\right)^{2} .
$$


Thus (3.10) yields, for all $t \in \Omega$,

$$
\left|\xi^{T} G(t)+\eta^{T} H(t)\right|^{2}+\left[\operatorname{dist}\left(\xi^{T} D(t), C^{\circ}\right)\right]^{2} \geq \tau|(\xi, \eta)|^{2} \quad\left((\xi, \eta) \in \mathbb{R}^{\gamma} \times \mathbb{R}^{\delta}\right) .
$$

Whence, by Theorem 3.1 and Remark 3.1, for each $t \in \Omega, H(t)$ is of full rank, and there exist two mappings $a_{t}: \mathbb{R}^{\gamma} \times \mathbb{R}^{\delta} \rightarrow \mathbb{R}^{m}$ and $c_{t}: \mathbb{R}^{\gamma} \times \mathbb{R}^{\delta} \rightarrow \mathbb{R}^{q}$ such that

$G(t) a_{t}(v, w)-D(t) c_{t}(v, w)=v, \quad H(t) a_{t}(v, w)=0, \quad c_{t}(v, w) \in C \quad\left((v, w) \in \mathbb{R}^{\gamma} \times \mathbb{R}^{\delta}\right)$,

$$
\left\|\left(H(t) H(t)^{T}\right)^{-1}\right\| \leq \rho,
$$

and

$$
\begin{aligned}
\left|a_{t}(v, w)\right| & \leq \rho\left[\|G\|_{\infty}+\|H\|_{\infty}\right]|(v, w)|, \\
\left|c_{t}(v, w)\right| & \leq \rho\|D\|_{\infty}|(v, w)| \quad\left((v, w) \in \mathbb{R}^{\gamma} \times \mathbb{R}^{\delta}\right) .
\end{aligned}
$$

Thus, with $a:=a_{t}(v, w)$ and $c:=c_{t}(v, w)$, we have that $(a, c) \in \Phi(t, v, w)$, whence the nonemptiness of $\Phi(t, v, w)$ follows.

Furthermore, $\Phi$ is $\mathcal{A} \times \mathcal{B} \times \mathcal{B}$-measurable with closed images. Hence, by the measurable selection theorem, there exists an $\mathcal{A} \times \mathcal{B} \times \mathcal{B}$-measurable function $(a, c)$ : $\Omega \times \mathbb{R}^{\gamma} \times \mathbb{R}^{\delta} \rightarrow \mathbb{R}^{m} \times \mathbb{R}^{q}$ such that

$$
(a(t, v, w), c(t, v, w)) \in \Phi(t, v, w) \quad\left((t, v, w) \in \Omega \times \mathbb{R}^{\gamma} \times \mathbb{R}^{\delta}\right) .
$$

Therefore, $c$ has nonnegative components and together with $a$ satisfies the relations (3.11) and (3.12), where $R:=\rho \max \left(\|G\|_{\infty}+\|H\|_{\infty},\|D\|_{\infty}\right)$.

Using (3.13), it follows that the function matrix-valued $B$ defined by $B(t)=$ $H^{T}(t)\left(H(t) H^{T}(t)\right)^{-1}$ is an essentially bounded right inverse of $H$.

4. Main results. We consider the optimal control problem

Minimize $\quad \ell(x(0), x(1))$

$$
\text { subject to }\left\{\begin{aligned}
\text { (i) } & a(x(0), x(1)) \in R, \\
\text { (ii) } & b(x(0), x(1))=0, \\
\text { (iii) } & \dot{x}(t)=f(t, x(t), u(t)) \text { for a.e. } t \in[0,1], \\
\text { (iv) } & g(t, x(t), u(t)) \in(t) \text { for a.e. } t \in[0,1], \\
\text { (v) } & h(t, x(t), u(t))=0 \text { for a.e. } t \in[0,1], \\
\text { (vi) } & k(t, x(t)) \in S(t) \text { for } t \in[0,1],
\end{aligned}\right.
$$

where $x:[0,1] \rightarrow \mathbb{R}^{n}$ is absolutely continuous, $u:[0,1] \rightarrow \mathbb{R}^{m}$ is essentially bounded measurable, and the ranges of the functions $\ell, a, b, f, g, h$, and $k$ are, respectively, in $\mathbb{R}, \mathbb{R}^{r}, \mathbb{R}^{s}, \mathbb{R}^{n}, \mathbb{R}^{\gamma}, \mathbb{R}^{\delta}$, and $\mathbb{R}^{\kappa}$. Furthermore, $R$ is a subset of $\mathbb{R}^{r}$, and $Q$ and $S$ are set-valued maps with images in $\mathbb{R}^{\gamma}$ and $\mathbb{R}^{\kappa}$.

The set-valued maps $Q$ and $S$ will be assumed in $\left(R_{5}\right)$ to take convex values, while no convexity is imposed on the functions $g$ and $k$. Hence, the forms of the constraints (iv) and (vi) considered here are more general than the traditional forms: $u(t) \in Q(t)$ and $x(t) \in S(t)$. Indeed, the present constraints permit us to consider, for instance, inequality constraints $g(t, x(t), u(t)) \leq 0$ and $k(t, x(t)) \leq 0$ without any convexity assumptions on the functions $g$ and $k$.

The Hamiltonian function associated to $(\mathcal{C P})$ is

$$
\mathcal{H}(t, x, u, p, \varphi, \psi):=p^{T} f(t, x, u)+\varphi^{T} g(t, x, u)+\psi^{T} h(t, x, u) .
$$


If $(x, u)$ satisfy (i)-(vi), then it is said to be admissible for (eP). Given an admissible arc $(\widehat{x}, \widehat{u})$, we denote by $\widehat{F}$ the evaluation of a given function $F$ along $(\widehat{x}, \widehat{u})$. For instance, $\widehat{a}:=a(\widehat{x}(0), \widehat{x}(1))$ and $\widehat{g}$ is defined by $\widehat{g}(t):=g(t, \widehat{x}(t), \widehat{u}(t))$.

To formulate the optimality concept and the regularity assumptions for problem (eP), introduce the following notion: If $T$ is a subset of $[0,1]$ and $\widehat{w}:[0,1] \rightarrow \mathbb{R}^{\omega}$ is an arbitrary function, then the $\varepsilon$-tube on $T$ around $\widehat{w}$ is the set

$$
\mathcal{T}_{\varepsilon}(\widehat{w} ; T):=\left\{(t, w) \in T \times \mathbb{R}^{\omega}|| w-\widehat{w}(t) \mid<\varepsilon \text { for } t \in T\right\} .
$$

When $T=\{t\}$ is a singleton, then $\left\{w \mid(t, w) \in \mathcal{T}_{\varepsilon}(\widehat{w} ;\{t\})\right\}$ will be denoted by $\mathcal{T}_{\varepsilon}(\widehat{w}(t))$.

A pair $(\widehat{x}, \widehat{u})$ provides a weak-local minimum for ( $\mathcal{C P})$ if there exists an $\varepsilon>0$ such that for all admissible pairs $(x, u) \in \mathcal{T}_{\varepsilon}(\widehat{x}, \widehat{u} ;[0,1])$, we have $\ell(x(0), x(1)) \geq$ $\ell(\widehat{x}(0), \widehat{x}(1))$.

In [OS95] and [MOS98] optimality conditions for the Pontryagin minimum were obtained in the absence of pure-state constraints and when the mixed state-control constraints take the form of equality and inequality.

Denote by $\mathcal{L}$ the class of Lebesgue-measurable subsets in $[0,1]$, and by $\mathcal{B}$ the class of Borel-measurable subsets in a metric space.

A pair $(\widehat{x}, \widehat{u})$ is called regular for (eP) if there exists an $\varepsilon>0$ such that the following conditions are satisfied:

$\left(R_{1}\right)$ The functions $\ell, a, b$ are defined on $\mathcal{T}_{\varepsilon}(\widehat{x} ;\{0,1\})$ and are strictly Fréchet differentiable at the point $(\widehat{x}(0), \widehat{x}(1))$.

$\left(R_{2}\right)$ The functions $f, g, h$ are defined on $\mathcal{T}_{\varepsilon}(\widehat{x}, \widehat{u} ;[0,1])$, are $\mathcal{L} \times \mathcal{B} \times \mathcal{B}$-measurable, and the maps

$$
\begin{aligned}
(x, u) & \mapsto f(t, x, u) \\
\text { and } \quad(x, u) & \mapsto(g(t, x, u), h(t, x, u)) \quad\left((x, u) \in \mathcal{T}_{\varepsilon}(\widehat{x}(t), \widehat{u}(t))\right)
\end{aligned}
$$

are strictly Fréchet differentiable at the point $(\widehat{x}(t), \widehat{u}(t)), \mathcal{L}^{1}$-uniformly and $\mathcal{L}^{\infty}$-uniformly, respectively, for a.e. $t \in[0,1]$. Furthermore, it is also assumed that $\widehat{f}, \widehat{f}_{x}$, and $\widehat{f}_{u}$ are integrable functions, and $\widehat{g}, \widehat{h}, \widehat{g}_{x}, \widehat{h}_{x}, \widehat{g}_{u}$, and $\widehat{h}_{u}$ are essentially bounded measurable functions.

$\left(R_{3}\right)$ The functions $g$ and $h$ satisfy the following strong normality condition: There exist a constant $\tau>0$ and bounded measurable functions $d_{1}, \ldots, d_{q} \in$ $T\left(\widehat{g} \mid \operatorname{sel}_{\infty}(Q)\right)$ such that, for a.e. $t \in[0,1]$,

$$
\left|\xi^{T} \widehat{g}_{u}(t)+\eta^{T} \widehat{h}_{u}(t)\right|^{2}+\sum_{i=1}^{q}\left[\left(\xi^{T} d_{i}(t)\right)^{+}\right]^{2} \geq \tau|(\xi, \eta)|^{2} \quad\left((\xi, \eta) \in \mathbb{R}^{\gamma} \times \mathbb{R}^{\delta}\right) .
$$

$\left(R_{4}\right)$ The function $k$ defined on $\mathcal{T}_{\varepsilon}(\widehat{x} ;[0,1])$ is Borel-measurable, and the map

$$
x \mapsto k(t, x) \quad\left(x \in \mathcal{T}_{\mathcal{\varepsilon}}(\widehat{x}(t))\right)
$$

is strictly Fréchet differentiable at the point $\widehat{x}(t)$ uniformly in $t \in[0,1]$. Furthermore, it is also assumed that $\widehat{k}$ and $\widehat{k}_{x}$ are continuous functions.

$\left(R_{5}\right)$ The set $R \subset \mathbb{R}^{r}$ is closed convex and has nonempty interior; the set-valued maps $Q:[0,1] \rightarrow 2^{\mathbb{R}^{\gamma}}$ and $S:[0,1] \rightarrow 2^{\mathbb{R}^{\kappa}}$ take closed convex values with nonempty interior and are measurable and lower semicontinuous, respectively. Moreover, $Q$ also satisfies condition (2.6). 
We note that, in $\left(R_{3}\right)$, a sufficient condition in order that $d_{1}, \ldots, d_{q} \in T\left(\widehat{g} \mid \operatorname{sel}_{\infty}(Q)\right)$ be valid is that $d_{1}, \ldots, d_{q} \in C\left(\widehat{g} \mid \operatorname{sel}_{\infty}(Q)\right)$ be satisfied. This latter condition holds if and only if $d_{1}(t), \ldots, d_{q}(t) \in C(\widehat{g}(t) \mid Q(t))$ almost uniformly in $t$, that is, if there exists a constant $M>0$ such that, for all $i=1, \ldots, q$ and for a.e. $t$,

$$
\left[\xi^{T} d_{i}(t)\right]^{2} \leq M|\xi|\left(\delta^{*}(\xi \mid Q(t))-\xi^{T} \widehat{g}(t)\right)
$$

whenever $\xi \in \mathbb{R}^{\gamma}$ satisfies $\xi^{T} d_{i}(t)>0$.

A pair $(\delta x, \delta u)$ is said to be critical for $(\mathcal{C P})$ at $(\widehat{x}, \widehat{u})$ if $\delta x:[0,1] \rightarrow \mathbb{R}^{n}$ is absolutely continuous, $\delta u:[0,1] \rightarrow \mathbb{R}^{m}$ is essentially bounded measurable, and

$\left(C_{1}\right) \widehat{\ell}_{x_{0}} \delta x(0)+\widehat{\ell}_{x_{1}} \delta x(1) \leq 0$

$\left(C_{2}\right) \widehat{a}_{x_{0}} \delta x(0)+\widehat{a}_{x_{1}} \delta x(1) \in C(\widehat{a} \mid R) ;$

$\left(C_{3}\right) \widehat{b}_{x_{0}} \delta x(0)+\widehat{b}_{x_{1}} \delta x(1)=0$

$\left(C_{4}\right) \dot{\delta} x(t)=\widehat{f}_{x}(t) \delta x(t)+\widehat{f}_{u}(t) \delta u(t)$ holds for a.e. $t \in[0,1]$;

$\left(C_{5}\right) \widehat{g}_{x}(t) \delta x(t)+\widehat{g}_{u}(t) \delta u(t) \in C(\widehat{g}(t) \mid Q(t))$ almost uniformly in $t \in[0,1]$; that is, there exists a constant $M>0$ such that, for a.e. $t \in[0,1]$,

$$
\left[\xi^{T} \widehat{g}_{x}(t) \delta x(t)+\xi^{T} \widehat{g}_{u}(t) \delta u(t)\right]^{2} \leq M|\xi|\left(\delta^{*}(\xi \mid Q(t))-\xi^{T} \widehat{g}(t)\right)
$$

whenever $\xi \in \mathbb{R}^{\gamma}$ satisfies $\xi^{T} \widehat{g}_{x}(t) \delta x(t)+\xi^{T} \widehat{g}_{u}(t) \delta u(t)>0 ;$

$\left(C_{6}\right) \widehat{h}_{x}(t) \delta x(t)+\widehat{h}_{u}(t) \delta u(t)=0$ holds for a.e. $t \in[0,1]$;

$\left(C_{7}\right) \widehat{k}_{x}(t) \delta x(t) \in C(\widehat{k}(t) \mid S(t))$ uniformly in $t \in[0,1]$; that is, there exists a constant $M>0$ such that, for all $t \in[0,1]$,

$$
\left[\zeta^{T} \widehat{k}_{x}(t) \delta x(t)\right]^{2} \leq M|\zeta|\left(\delta^{*}(\zeta \mid S(t))-\zeta^{T} \widehat{k}(t)\right)
$$

whenever $\zeta \in \mathbb{R}^{\kappa}$ satisfies $\zeta^{T} \widehat{k}_{x}(t) \delta x(t)>0$.

A critical arc $(\delta x, \delta u)$ is called regular for $(\mathrm{eP})$ at $(\widehat{x}, \widehat{u})$ if

$\left(R_{6}\right) \hat{\ell}, \widehat{a}$, and $\widehat{b}$ are twice directionally differentiable at $(\widehat{x}(0), \widehat{x}(1))$ in direction $(\delta x(0), \delta x(1))$

$\left(R_{7}\right)$ for a.e. $t \in[0,1]$, the maps in (4.1) are twice directionally differentiable at $(\widehat{x}(t), \widehat{u}(t))$ in direction $(\delta x(t), \delta u(t)) \mathcal{L}^{1}$ - and $\mathcal{L}^{\infty}$-uniformly in $t$, respectively;

$\left(R_{8}\right)$ for all $t \in[0,1]$, the map (4.3) is twice directionally differentiable at $\widehat{x}(t)$ in direction $\delta x(t)$ uniformly in $t$.

The following result consists of necessary conditions for optimality in (eP). Its proof makes use of all the results of sections 2 and 3 and applies the argument followed in [PZ94b].

THEOREM 4.1. Let $(\widehat{x}, \widehat{u})$ be a regular weak local minimum for the problem (eP). Then, for every regular critical arc $(\delta x, \delta u)$, there exist constants $\lambda \in \mathbb{R}$, $\alpha=\left(\alpha_{1}, \ldots, \alpha_{r}\right) \in \mathbb{R}^{r}, \beta=\left(\beta_{1}, \ldots, \beta_{s}\right) \in \mathbb{R}^{s}$, an absolutely continuous function $p:[0,1] \rightarrow \mathbb{R}^{n}$, two integrable functions $\varphi:[0,1] \rightarrow \mathbb{R}^{\gamma}$ and $\psi:[0,1] \rightarrow \mathbb{R}^{\delta}$, and a Borel regular vector-valued measure $\mu=\left(\mu_{1}, \ldots, \mu_{\kappa}\right)$, not all zero, such that $\lambda \geq 0$,

$$
\alpha \in N(\widehat{a} \mid R), \quad \alpha^{T}\left(\widehat{a}_{x_{0}} \delta x(0)+\widehat{a}_{x_{1}} \delta x(1)\right)=0,
$$

$$
\begin{aligned}
& \varphi(t) \in N(\widehat{g}(t) \mid Q(t)), \quad \varphi^{T}(t)\left(\widehat{g}_{x}(t) \delta x(t)+\widehat{g}_{u} \delta u(t)\right)=0 \quad \text { for a.e. } t \in[0,1], \\
& \frac{d \mu}{d|\mu|}(t) \in N(\widehat{k}(t) \mid S(t)), \quad\left(\frac{d \mu}{d|\mu|}\right)^{T}(t) \widehat{k}_{x}(t) \delta x(t)=0 \quad \text { for } \mu \text {-a.e. } t \in[0,1]
\end{aligned}
$$




$$
\begin{gathered}
\dot{p}^{T}(t)=-\widehat{\mathcal{H}}_{x}\left(t, p(t)+\int_{] t, 1]} \widehat{k}_{x}^{T}(s) d \mu(s), \varphi(t), \psi(t)\right) \quad \text { for a.e. } t \in[0,1], \\
-p^{T}(0)=\lambda \widehat{\ell}_{x_{0}}+\alpha^{T} \widehat{a}_{x_{0}}+\beta^{T} \widehat{b}_{x_{0}}+\left(\int_{[0,1]} \widehat{k}_{x}^{T}(t) d \mu(t)\right)^{T}, \\
p^{T}(1)=\lambda \widehat{\ell}_{x_{1}}+\alpha^{T} \widehat{a}_{x_{1}}+\beta^{T} \widehat{b}_{x_{1}}, \\
\widehat{\mathcal{H}}_{u}\left(t, p(t)+\int_{] t, 1]} \widehat{k}_{x}^{T}(s) d \mu(s), \varphi(t), \psi(t)\right)=0 \quad \text { for a.e. } t \in[0,1],
\end{gathered}
$$

and

$$
\begin{aligned}
&\left(\lambda \widehat{\ell}^{\prime \prime}+\alpha^{T} \widehat{a}^{\prime \prime}+\beta^{T} \widehat{b}^{\prime \prime}\right)(\delta x(0), \delta x(1))+\int_{0}^{1} \widehat{k}^{\prime \prime}(t ; \delta x(t)) d \mu(t) \\
&+\int_{0}^{1} \widehat{\mathcal{H}}^{\prime \prime}\left(t, p(t)+\int_{] t, 1]} \widehat{k}_{x}^{T}(s) d \mu(s), \varphi(t), \psi(t) ; \delta x(t), \delta u(t)\right) d t \\
& \geq 2 \overline{\operatorname{co}} \mathcal{E}\left(\widehat{a}, \widehat{a}^{\prime}(\delta x(0), \delta x(1)) \mid R\right)(\alpha)+2 \int_{0}^{1} \overline{\operatorname{co}} \mathbb{E}\left(\widehat{k}, \widehat{k}_{x} \delta x \mid S\right)\left(t, \frac{d \mu}{d|\mu|}(t)\right) d|\mu|(t) \\
&+2 \int_{0}^{1} \overline{\operatorname{co}} \mathcal{E}\left(\widehat{g}(t), \widehat{g}_{x}(t) \delta x(t)+\widehat{g}_{u}(t) \delta u(t) \mid Q(t)\right)(\varphi(t)) d t,
\end{aligned}
$$

where $\mathcal{H}^{\prime \prime}$ denotes the second-order strong directional derivative of $\mathcal{H}$ with respect to the variable $(x, u)$.

Proof. First we are going to apply the result of Theorem 2.5, which is a special case of [PZ94b, Theorem 3]. Introduce the following spaces

$$
\begin{gathered}
X:=\mathcal{C}\left(\mathbb{R}^{n}\right), \quad U:=\mathcal{L}_{m}^{\infty}:=\mathcal{L}^{\infty}\left(\mathbb{R}^{m}\right), \quad Y:=\mathbb{R}^{s} \times\left\{y \in \mathcal{C}\left(\mathbb{R}^{n}\right) \mid y(0)=0\right\}, \\
V:=\mathbb{R}^{r} \times \mathcal{C}\left(\mathbb{R}^{\kappa}\right) \times \mathcal{L}_{\gamma}^{\infty}, \quad W:=\mathcal{L}_{\delta}^{\infty}
\end{gathered}
$$

(where we suppress $[0,1]$ in this notation) and denote, for $(x, u) \in X \times U$,

$$
\begin{aligned}
\mathbf{F}(x, u) & :=\ell(x(0), x(1)), \\
\mathbf{G}(x, u)(t) & :=\left(\begin{array}{l}
a(x(0), x(1)) \\
k(t, x(t)) \\
g(t, x(t), u(t))
\end{array}\right), \\
\mathbf{H}(x, u)(t) & :=h(t, x(t), u(t)), \\
\mathbf{K}(x, u)(t) & :=\left(\begin{array}{c}
b(x(0), x(1)) \\
\int_{0}^{t}(f(\tau, x(\tau), u(\tau)) d \tau-x(t)+x(0),
\end{array}\right), \\
\mathbf{Q} & :=R \times \operatorname{sel}_{C}(S) \times \operatorname{sel}_{\infty}(Q) .
\end{aligned}
$$


Then, with this notation, our control problem (eP) is equivalent to the abstract control problem $(\mathcal{P})$ in section 2.

The regularity condition $\left(R_{5}\right)$ yields that the set $\mathbf{Q}$ defined above is closed, convex, with nonempty interior.

Let $\varepsilon>0$ be the constant for which the regularity assumptions of the arc $(\widehat{x}, \widehat{u})$ are satisfied and define

$$
D:=\left\{(x, u) \in X \times U \mid\|x-\widehat{x}\|<\varepsilon,\|u-\widehat{u}\|_{\infty}<\varepsilon\right\} .
$$

Then $D$ is an open subset of $X \times U$, and the functions $\mathbf{F}, \mathbf{G}, \mathbf{H}, \mathbf{K}$ are defined on $D$. Since the $\operatorname{arc}(\widehat{x}, \widehat{u})$ satisfies the regularity conditions $\left(R_{1}\right),\left(R_{2}\right)$, and $\left(R_{4}\right)$ for $(\mathrm{CP})$, the functions $\mathbf{F}, \mathbf{G}, \mathbf{H}$, and $\mathbf{K}$ are strictly Fréchet differentiable at $(\widehat{x}, \widehat{u})$, whence we have the following relations:

$$
\widehat{\mathbf{H}}^{\prime}(x, u)(t)=\widehat{H}_{x}(t) x(t)+\widehat{H}_{u}(t) u(t)
$$

and

$$
\begin{aligned}
\widehat{\mathbf{K}}_{u}(x, u)(t) & :=\left(\begin{array}{l}
0 \\
\int_{0}^{t}\left(\widehat{f}_{u}(\tau) u(\tau) d \tau\right.
\end{array}\right), \\
\widehat{\mathbf{K}}_{x}(x, u)(t) & :=\left(\begin{array}{c}
\widehat{b}(x(0), x(1)) \\
\int_{0}^{t}\left(\widehat{f}_{x}(\tau) x(\tau) d \tau-x(t)+x(0)\right.
\end{array}\right) .
\end{aligned}
$$

We need to show that the partial Fréchet derivatives $\widehat{\mathbf{K}}_{x}$ and $\widehat{\mathbf{K}}_{u}$ of the mapping $\mathbf{K}$ are Fredholm and compact operators, respectively. Since $\mathbf{K}_{u}$ is a Volterra integral operator, it is compact. On the other hand, the operator $\mathbf{K}_{x}$ is the sum of a compact (Volterra integral) operator and the operator $F: X \rightarrow Y$ defined by $F x(t):=-x(t)+$ $x(a)$, which is clearly a Fredholm operator. Therefore, by [PZ94b, Lemmas 3 and 5], $\mathbf{K}_{x}$ is also Fredholm. Thus, for $\left(\mathbf{R}_{1}\right)-\left(\mathbf{R}_{3}\right)$ to hold, it remains to show that $\widehat{\mathbf{H}}_{u}$ has a bounded right inverse. The strong normality condition, i.e., $\left(R_{3}\right)$, and Theorem 3.2 yield that the function $\widehat{h}_{u} \widehat{h}_{u}^{T}:[0,1] \rightarrow \mathbb{R}^{\delta}$ has a bounded measurable inverse, and hence the linear operator $\mathbf{B}: W \rightarrow U$ defined by

$$
(\mathbf{B} w)(t):=\widehat{h}_{u}^{T}(t)\left(\widehat{h}_{u}(t) \widehat{h}_{u}^{T}(t)\right)^{-1} w(t) \quad(t \in[0,1])
$$

is a bounded linear right inverse for $\mathbf{H}_{u}(\widehat{x}, \widehat{u})$.

Hence, the $\operatorname{arc}(\widehat{x}, \widehat{u})$ is a regular arc with respect to the problem $(\mathcal{P})$.

Now we prove that the pair $(\delta x, \delta u)$ is a regular and critical arc for $(\mathcal{P})$ at the point $(\widehat{x}, \widehat{u})$ where $\mathbf{F}, \mathbf{G}, \mathbf{H}$, and $\mathbf{K}$ are defined above. The regularity assumptions $\left(R_{6}\right)-\left(R_{8}\right)$ imposed on $(\delta x, \delta u)$ yield that the functions $\mathbf{F}, \mathbf{G}, \mathbf{H}$, and $\mathbf{K}$ are twice directionally differentiable at $(\widehat{x}, \widehat{u})$ in the direction $(\delta x, \delta u)$; that is, $\left(\mathbf{R}_{4}\right)$ holds. Note that $\left(C_{1}\right)$ implies $\left(\mathbf{C}_{1}\right)$. Using $\left(C_{7}\right)$ together with Theorem 2.1, $\left(C_{5}\right)$ together with Theorem 2.2, and $\left(C_{2}\right)$, then applying the product rule, we can see that

$$
\widehat{\mathbf{G}}_{x} \delta x+\widehat{\mathbf{G}}_{u} \delta u \in C(\widehat{\mathbf{G}} \mid \mathbf{Q}) ;
$$

that is, the second-order variation set $V\left(\widehat{\mathbf{G}}, \widehat{\mathbf{G}}_{x} \delta x+\widehat{\mathbf{G}}_{u} \delta u \mid \mathbf{Q}\right)$ is nonempty. Furthermore, $\left(C_{3}\right),\left(C_{4}\right)$, and $\left(C_{6}\right)$ yield that

$$
\widehat{\mathbf{H}}_{x} \delta x+\widehat{\mathbf{H}}_{u} \delta u=0, \quad \widehat{\mathbf{K}}_{x} \delta x+\widehat{\mathbf{K}}_{u} \delta u=0 .
$$


Then $\left(\mathbf{C}_{2}\right)$ is satisfied, and hence $(\delta x, \delta u)$ is a regular and critical arc for $(\mathcal{P})$ at the point $(\widehat{x}, \widehat{u})$.

Therefore, the statement of Theorem 2.5 can also be applied to produce multipliers $\lambda \geq 0, v^{*}=\left(v_{1}^{*}, v_{2}^{*}, v_{3}^{*}\right) \in V^{*}, w^{*} \in W^{*}$, and $y^{*}=\left(y_{1}^{*}, y_{2}^{*}\right) \in Y^{*}$, not all zero, satisfying (2.16)-(2.19). The first and second components $v_{1}^{*}$ and $v_{2}^{*}$ of $v^{*}$ can be identified, respectively, by a vector $\alpha \in \mathbb{R}^{r}$ and (due to the Riesz representation theorem) by a bounded signed $\mathbb{R}^{\kappa}$-valued Borel measure $\mu$. For the third component, we have $v_{3}^{*} \in\left(\mathcal{L}_{\gamma}^{\infty}\right)^{*}$. Then $(2.16)$ yields that

$$
\left(\alpha, \mu, v_{3}^{*}\right) \in N(\widehat{\mathbf{G}} \mid \mathbf{Q})=N(\widehat{a} \mid R) \times N\left(\widehat{k} \mid \operatorname{sel}_{C}(S)\right) \times N\left(\widehat{g} \mid \operatorname{sel}_{\infty}(Q)\right)
$$

and

$$
\alpha^{T}\left(\widehat{a}_{x_{0}} \delta x(0)+\widehat{a}_{x_{1}} \delta x(1)\right)+\int_{[0,1]}\left(\widehat{k}_{x}(t) \delta x(t)\right)^{T} d \mu(t)+\left\langle v_{3}^{*}, \widehat{g}_{x} \delta x+\widehat{g}_{u} \delta u\right\rangle=0 .
$$

Therefore, we get that the first equation of (4.7) is valid and that $\mu \in N\left(\widehat{k} \mid \operatorname{sel}_{C}(S)\right)$, which, via (2.2), yields the first equation of (4.9); furthermore,

$$
v_{3}^{*} \in N\left(\widehat{g} \mid \operatorname{sel}_{\infty}(Q)\right) .
$$

The first component $y_{1}^{*}$ of $y^{*}$ can be identified by an element $\beta \in \mathbb{R}^{s}$, and, by the Riesz representation theorem, there exists a bounded signed $\mathbb{R}^{n}$-valued measure $\nu$ with $\nu(\{0\})=0$ such that $y_{2}^{*}$ is represented via $\nu$; that is, for $y \in \mathcal{C}\left(\mathbb{R}^{n}\right)$ with $y(0)=0$, we have

$$
\left\langle y_{2}^{*}, y\right\rangle=\int_{[0,1]} y^{T}(t) d \nu(t)
$$

Define $\bar{p}:[0,1] \rightarrow \mathbb{R}^{n}$ by

$$
\bar{p}(t)=\nu(] t, 1]) .
$$

Clearly, $\bar{p}(1)=0$, and $\bar{p}$ is of bounded variation (and hence it is also bounded). Then, by standard argument (see, e.g., [PZ94b, p. 441]), we get that, for $x \in \mathcal{C}\left(\mathbb{R}^{n}\right)$ and $u \in \mathcal{L}_{m}^{\infty}$,

$\left\langle y^{*}, \widehat{\mathbf{K}}_{x} x\right\rangle=\beta^{T}\left(\widehat{b}_{x_{0}} x(0)+\widehat{b}_{x_{1}} x(1)\right)+\int_{0}^{1} \bar{p}^{T}(t) \widehat{f}_{x}(t) x(t) d t-\int_{0}^{1} x^{T}(t) d \nu(t)+\bar{p}^{T}(0) x(0)$,

$$
\left\langle y^{*}, \widehat{\mathbf{K}}_{u} u\right\rangle=\int_{0}^{1} \bar{p}^{T}(t) \widehat{f}_{u}(t) u(t) d t
$$

and

$$
\left\langle y^{*}, \widehat{\mathbf{K}}^{\prime \prime}(\delta x, \delta u)\right\rangle=\beta^{T} \widehat{b}^{\prime \prime}(\delta x(0)+\delta x(1))+\int_{0}^{1} \bar{p}^{T}(t) \widehat{f}^{\prime \prime}(t ; \delta x(t), \delta u(t)) d t .
$$

Using (4.18), equation (2.18) reduces to

$$
\left\langle v_{3}^{*}, \widehat{g}_{u} u\right\rangle+\left\langle w^{*}, \widehat{h}_{u} u\right\rangle+\int_{0}^{1} \bar{p}^{T}(t) \widehat{f}_{u}(t) u(t) d t=0 \quad\left(u \in \mathcal{L}_{m}^{\infty}\right) .
$$


We are going to show that $v_{3}^{*}$ and $w$ are represented via integrable functions. To achieve this goal, we shall apply Theorem 3.2. Observe that condition (4.2) is equivalent to (3.10), where $G$ and $H$ are replaced by $\widehat{g}_{u}$ and $\widehat{h}_{u}$, respectively, and $d_{1}, \ldots, d_{q}$ are the functions of hypothesis $\left(R_{3}\right)$ of the theorem. Thus, by Theorem 3.2, $\widehat{h}_{u} \widehat{h}_{u}^{T}$ has a bounded measurable inverse and there exist $\mathcal{L} \times \mathcal{B} \times \mathcal{B}$-measurable functions $a:[0,1] \times \mathbb{R}^{\gamma} \times \mathbb{R}^{\delta} \rightarrow \mathbb{R}^{m}, c_{1}, \ldots, c_{q}:[0,1] \times \mathbb{R}^{\gamma} \times \mathbb{R}^{\delta} \rightarrow[0, \infty[$ and a constant $R>0$ such that, for a.e. $t \in[0,1]$,

$\widehat{g}_{u}(t) a(t, v, w)=v+\sum_{i=1}^{q} c_{i}(t, v, w) d_{i}(t), \quad \widehat{h}_{u}(t) a(t, v, w)=w \quad\left((v, w) \in \mathbb{R}^{\gamma} \times \mathbb{R}^{\delta}\right)$,

and

$|a(t, v, w)| \leq R|(v, w)|, \quad c_{i}(t, v, w) \leq R|(v, w)| \quad\left((v, w) \in \mathbb{R}^{\gamma} \times \mathbb{R}^{\delta}, i=1, \ldots, q\right)$.

Let $(v, w) \in \mathcal{L}_{\gamma}^{\infty} \times \mathcal{L}_{\delta}^{\infty}$ be fixed. Set

$$
\begin{aligned}
\mathbb{A}(v, w)(t) & :=\boldsymbol{a}(t):=a(t, v(t), w(t)), \\
\boldsymbol{c}_{i}(t) & :=c_{i}(t, v(t), w(t)) \quad(t \in[0,1], i=1, \ldots, q) .
\end{aligned}
$$

Then, due to the second inequality in (4.22), $\boldsymbol{a}$ and $\boldsymbol{c}_{i}$ are bounded measurable functions. Thus $\mathbb{A}$ is defined on $\mathcal{L}_{\gamma}^{\infty} \times \mathcal{L}_{\delta}^{\infty}$ with a range in $\mathcal{L}_{m}^{\infty}$. Since $\operatorname{sel}_{\infty}(Q)$ is decomposable, $T\left(\widehat{g} \mid \operatorname{sel}_{\infty}(Q)\right)$ is an $\mathcal{L}$-cone, and we have that

$$
\sum_{i=1}^{q} \boldsymbol{c}_{i} d_{i} \in T\left(\widehat{g} \mid \operatorname{sel}_{\infty}(Q)\right) .
$$

Hence, by (4.21),

$$
\widehat{g}_{u} \mathbb{A}(v, w)-v \in T\left(\widehat{g} \mid \operatorname{sel}_{\infty}(Q)\right), \quad \widehat{h}_{u} \mathbb{A}(v, w)=w \quad\left((v, w) \in \mathcal{L}_{\gamma}^{\infty} \times \mathcal{L}_{\delta}^{\infty}\right) .
$$

Using (4.16), the first inclusion in (4.23) yields that

$$
\left\langle v_{3}^{*}, \widehat{g}_{u} \mathbb{A}(v, w)\right\rangle \leq\left\langle v_{3}^{*}, v\right\rangle \quad\left((v, w) \in \mathcal{L}_{\gamma}^{\infty} \times \mathcal{L}_{\delta}^{\infty}\right) .
$$

Now, substituting $u=\mathbb{A}(v, w)$ into (4.20) and using also the second relation in (4.23), we get that

$$
\left\langle v_{3}^{*}, v\right\rangle+\left\langle w^{*}, w\right\rangle+\int_{0}^{1} \bar{p}^{T}(t) \widehat{f}_{u}(t) a(t, v(t), w(t)) d t \geq 0 \quad\left((v, w) \in \mathcal{L}_{\gamma}^{\infty} \times \mathcal{L}_{\delta}^{\infty}\right) .
$$

Putting $w=0$, we deduce that

$$
\left|\left\langle v_{3}^{*}, v\right\rangle\right| \leq\left|\int_{0}^{1} \bar{p}(t) \widehat{f}_{u}(t) a(t, v(t), 0) d t\right| \leq \int_{0}^{1}|\bar{p}(t)|\left|\widehat{f}_{u}(t)\right| R|v(t)| d t \quad\left(v \in \mathcal{L}_{\gamma}^{\infty}\right) .
$$

Hence, $v_{3}^{*}$ is $\mathcal{L}^{1}$-continuous; i.e., for any bounded (in $\left.\mathcal{L}_{\gamma}^{\infty}\right)$ sequence $\left(v_{i}\right)$ that converges almost everywhere to zero, we have that $\left\langle v_{3}^{*}, v_{i}\right\rangle$ tends to zero. Therefore, by the 
Yosida-Hewitt representation theorem, there exists an integrable function $\varphi:[0,1] \rightarrow$ $\mathbb{R}^{\gamma}$ such that

$$
\left\langle v_{3}^{*}, v\right\rangle=\int_{0}^{1} \varphi^{T}(t) v(t) d t \quad\left(v \in \mathcal{L}_{\gamma}^{\infty}\right) .
$$

Arguing analogously for $w^{*},(4.24)$ also yields the existence of an integrable function $\psi:[0,1] \rightarrow \mathbb{R}^{\delta}$ such that

$$
\left\langle w^{*}, w\right\rangle=\int_{0}^{1} \psi^{T}(t) w(t) d t \quad\left(w \in \mathcal{L}_{\delta}^{\infty}\right) .
$$

By (2.5), we have that (4.16) is equivalent to the first equation of (4.8). Furthermore, (4.15) and conditions $\left(C_{2}\right),\left(C_{5}\right)$, and $\left(C_{7}\right)$ combined with the first equations of (4.7)(4.9) yield the second equations of (4.7)-(4.9).

Using the representations of $v_{3}^{*}$ and $w^{*}$ and (4.17), equation (2.17) can be rewritten in the following way:

For all $x \in \mathcal{C}\left(\mathbb{R}^{n}\right)$,

$$
\begin{aligned}
& \left(\lambda \widehat{l}_{x_{0}}+\alpha^{T} \widehat{a}_{x_{0}}+\beta^{T} \widehat{b}_{x_{0}}+\bar{p}^{T}(0)\right) x(0)+\left(\lambda \widehat{l}_{x_{1}}+\alpha^{T} \widehat{a}_{x_{1}}+\beta^{T} \widehat{b}_{x_{1}}\right) x(1)-\int_{0}^{1} x^{T}(t) d \nu(t) \\
& +\int_{0}^{1} x^{T}(t) \widehat{k}_{x}^{T}(t) d \mu(t)+\int_{0}^{1}\left[\varphi^{T}(t) \widehat{g}_{x}(t)+\psi^{T}(t) \widehat{h}_{x}(t)+\bar{p}^{T}(t) \widehat{f}_{x}(t)\right] x(t) d t=0 .
\end{aligned}
$$

Set

$$
p(t):= \begin{cases}\bar{p}(t)-\int_{] t, 1]} \widehat{k}_{x}^{T}(t) d \mu(t) & \text { for } t \in[0,1[, \\ \lim _{t \rightarrow 1-} p(t)=\nu(\{1\})-\widehat{k}_{x}^{T}(1) \mu(\{1\}) & \text { for } t=1 .\end{cases}
$$

Observe that (4.27) is also true for all functions $x$ of the form $x(t)=\bar{x} \chi_{\Omega}(t)$, where $\Omega$ is a subinterval of $[0,1]$ and $\bar{x} \in \mathbb{R}^{n}$ is arbitrary.

First taking $\Omega:=\{1\}$, it follows from (4.27) that

$$
\left(\lambda \widehat{l}_{x_{1}}+\alpha^{T} \widehat{a}_{x_{1}}+\beta^{T} \widehat{b}_{x_{1}}\right)^{T}-\nu(\{1\})+\widehat{k}_{x}^{T}(1) \mu(\{1\})=0 ;
$$

hence

$$
p(1)=\nu(\{1\})-\widehat{k}_{x}^{T}(1) \mu(\{1\})=\left(\lambda \widehat{l}_{x_{1}}+\alpha^{T} \widehat{a}_{x_{1}}+\beta^{T} \widehat{b}_{x_{1}}\right)^{T},
$$

which is exactly (4.12).

With the substitution $x(t):=\bar{x} \chi_{\{0\}}(t)\left(\bar{x} \in \mathbb{R}^{n}\right)$, we deduce from (4.27) that

$$
\lambda \widehat{l}_{x_{0}}+\alpha^{T} \widehat{a}_{x_{0}}+\beta^{T} \widehat{b}_{x_{0}}+p^{T}(0)+\left(\int_{] 0,1]} \widehat{k}_{x}^{T}(t) d \mu(t)+\widehat{k}_{x}^{T}(0) \mu(\{0\})\right)^{T}=0
$$

since $\nu(\{0\})=0$. This yields (4.11).

Finally, we put $x(t):=\bar{x} \chi_{] \tau, 1]}(t)$ into (4.27), where $\bar{x} \in \mathbb{R}^{n}$ and $\tau \in[0,1[$ are fixed arbitrarily. Then we obtain, for all $\tau \in[0,1[$, that

$$
\begin{aligned}
\lambda \widehat{l}_{x_{1}}+\alpha^{T} \widehat{a}_{x_{1}} & \left.\left.+\beta^{T} \widehat{b}_{x_{1}}+\left(\int_{] \tau, 1]} \widehat{k}_{x}^{T}(t) d \mu(t)-\nu(] \tau, 1\right]\right)\right)^{T} \\
& +\int_{\tau}^{1}\left[\varphi^{T}(t) \widehat{g}_{x}(t)+\psi^{T}(t) \widehat{h}_{x}(t)+\bar{p}^{T}(t) \widehat{f}_{x}(t)\right] d t=0 .
\end{aligned}
$$


Using (4.12) and the definitions of $p, \bar{p}$, and the Hamiltonian $\mathcal{H}$, the above equation can be rewritten as

$$
p^{T}(\tau)=p^{T}(1)+\int_{\tau}^{1} \mathcal{H}_{x}\left(t, p(t)+\int_{] t, 1]} \widehat{k}_{x}^{T}(s) d \mu(s), \varphi(t), \psi(t)\right) d t \quad(\tau \in[0,1[) .
$$

It follows from the above equation that $p$ is absolutely continuous, and after differentiation, we obtain (4.10).

Now we consider (2.18). Using (4.18), (4.25), and (4.26), equation (2.18) can be rewritten as

$$
\int_{0}^{1}\left[\varphi^{T}(t) \widehat{g}_{u}(t)+\psi^{T}(t) \widehat{h}_{u}(t)+\bar{p}^{T}(t) \widehat{f}_{u}(t)\right] u(t) d t=0 \quad\left(u \in \mathcal{L}_{m}^{\infty}\right)
$$

This is equivalent to

$$
\int_{0}^{1} \mathcal{H}_{u}\left(t, p(t)+\int_{] t, 1]} \widehat{k}_{x}^{T}(s) d \mu(s), \varphi(t), \psi(t)\right) u(t) d t=0 \quad\left(u \in \mathcal{L}_{m}^{\infty}\right) .
$$

By a standard argument, the above equation yields (4.13).

Now, (2.19) becomes

$$
\begin{aligned}
&\left(\lambda \widehat{l}^{\prime \prime}+\lambda^{T} \widehat{a}^{\prime \prime}+\beta^{T} \widehat{b}^{\prime \prime}\right)(\delta x(0), \delta x(1))+\int_{0}^{1} \widehat{k}^{\prime \prime}(t ; \delta x(t)) d \mu(t) \\
&+\int_{0}^{1} \mathcal{H}^{\prime \prime}\left(t, p(t)+\int_{] t, 1]} \widehat{k}_{x}^{T}(s) d \mu(s), \varphi(t), \psi(t) ; \delta x(t), \delta u(t)\right) d t \\
& \geq 2 \delta^{*}\left(v^{*} \mid V\left(\widehat{\mathbf{G}}, \widehat{\mathbf{G}}_{x} \delta x+\widehat{\mathbf{G}}_{u} \delta u \mid \mathbf{Q}\right)\right) \\
&=2 \delta^{*}\left(\alpha \mid V\left(\widehat{a}, \widehat{a}_{x_{0}} \delta x(0)+\widehat{a}_{x_{1}} \delta x(1) \mid R\right)\right)+2 \delta^{*}\left(\mu \mid V\left(\widehat{k}, \widehat{k}_{x} \delta x \mid \operatorname{sel}_{C}(S)\right)\right) \\
&+2 \delta^{*}\left(v_{3}^{*} \mid V\left(\widehat{g}, \widehat{g}_{x} \delta x+\widehat{g}_{u} \delta u \mid \operatorname{sel}_{\infty}(Q)\right)\right)
\end{aligned}
$$

since $\mathbf{Q}$ is the Cartesian product of three sets and therefore the sum rule applies. Now, the second and third terms on the right-hand side can be computed via Theorems 2.4 and 2.3, respectively. The first term can also be calculated via Theorem 2.5 , where the measure space $\Omega$ is chosen to be the singleton $\{0\}$ with $\mathcal{A}=\{\{0\}\}, \nu(\{0\})=1$. Thus the above inequality yields (4.14).

Now we consider a special case $(\widetilde{\mathcal{C P}})$ of problem $(\mathcal{C P})$, where

$$
R=\mathbb{R}_{-}^{r}, \quad \text { and } \quad Q(t)=\mathbb{R}_{-}^{\gamma}, \quad S(t)=\mathbb{R}_{-}^{\kappa} \quad \text { for all } \quad t \in[0,1] .
$$

In this case, we intend to simplify the results given by Theorem 4.1. Then $\left(R_{5}\right)$ is automatically satisfied. The focus is on reformulating conditions $\left(R_{3}\right),\left(C_{2}\right),\left(C_{5}\right)$, and $\left(C_{7}\right)$, and, in Theorem 4.1, conditions (4.7), (4.8), (4.9), and (4.14).

Condition $\left(R_{3}\right)$ is replaced by the following:

$\left(\widetilde{R}_{3}\right)$ There exist bounded measurable functions $d_{1}, \ldots, d_{q} \in \mathcal{L}_{\gamma}^{\infty}$ and a constant $M$ such that, for almost all $t \in[0,1]$ and for all $i=1, \ldots, q, j=1, \ldots, \gamma$,

$$
\begin{array}{cc}
\frac{d_{i j}^{2}(t)}{\widehat{g}_{j}(t)}>-M \quad \text { whenever } \quad & \widehat{g}_{j}(t)<0 \text { and } d_{i j}(t)>0, \\
d_{i j}(t) \leq 0 \quad \text { whenever } & \widehat{g}_{j}(t)=0 .
\end{array}
$$

Furthermore, there exists a constant $\tau>0$ such that, for a.e. $t \in[0,1],(4.2)$ is satisfied. 
Remark 4.1. It follows from Corollary 2.1 that if $d_{1}, \ldots, d_{q}$ satisfy (4.29) and (4.30), then $d_{1}, \ldots, d_{q} \in C\left(\widehat{g} \mid \operatorname{sel}_{\infty}(Q)\right)$; therefore, they also belong to $T\left(\widehat{g} \mid \operatorname{sel}_{\infty}(Q)\right)$.

Taking the choice $q=2 \gamma, d_{i}=\left(d_{i, 1}, \ldots, d_{i, \gamma}\right)$, where $d_{i, j}$ is defined by

$d_{i, j}=\left\{\begin{array}{ll}\sqrt{-\widehat{g}_{i}}, & i=j, \\ 0, & i \neq j,\end{array} \quad d_{\gamma+i, j}=\left\{\begin{array}{ll}-\sqrt{-\widehat{g}_{i}}, & i=j, \\ 0, & i \neq j\end{array} \quad(i, j=1, \ldots, \gamma)\right.\right.$,

we can see that $d_{1}, \ldots, d_{2 \gamma}$ satisfy (4.29) and (4.30). In this case, (4.2) is equivalent to the following quadratic inequality: for a.e. $t \in[0,1]$,

$$
\left|\xi^{T} \widehat{g}_{u}(t)+\eta^{T} \widehat{h}_{u}(t)\right|^{2}-\sum_{i=1}^{\gamma} \xi_{i}^{2} \widehat{g}_{i}(t) \geq \tau|(\xi, \eta)|^{2} \quad\left((\xi, \eta) \in \mathbb{R}^{\gamma} \times \mathbb{R}^{\delta}\right) .
$$

Introducing the notation

$$
J(t):=\left(\begin{array}{cccc}
\widehat{g}_{1 u}(t) & \sqrt{-\widehat{g}_{1}(t)} & \ldots & 0 \\
\vdots & \vdots & \ddots & \vdots \\
\widehat{g}_{\gamma u}(t) & 0 & \cdots & \sqrt{-\widehat{g}_{\gamma}(t)} \\
\widehat{h}_{1 u}(t) & 0 & \cdots & 0 \\
\vdots & \vdots & \ddots & \vdots \\
\widehat{h}_{\delta u}(t) & 0 & \cdots & 0
\end{array}\right),
$$

we can rewrite (4.31) as

$$
\left(\xi^{T}, \eta^{T}\right) J(t) J^{T}(t)\left(\begin{array}{c}
\xi \\
\eta
\end{array}\right) \geq \tau|(\xi, \eta)|^{2} \quad\left((\xi, \eta) \in \mathbb{R}^{\gamma} \times \mathbb{R}^{\delta}\right) .
$$

Therefore, it is necessary and sufficient that

$$
\operatorname{det}\left(J(t) J^{T}(t)\right) \geq \tau \quad \text { for a.e. } t \in[0,1] .
$$

This latter condition appeared among the assumptions of Theorem 5 in [PZ94b].

The conditions $\left(C_{2}\right),\left(C_{5}\right)$, and $\left(C_{7}\right)$ are replaced by the following:

$\left(\widetilde{C}_{2}\right) \widehat{a}_{i, x_{0}} \delta x(0)+\widehat{a}_{i, x_{1}} \delta x(1) \leq 0$ whenever $\widehat{a}_{i}=0(i=1, \ldots, r)$.

$\left(\widetilde{C}_{5}\right)$ For all $i=1, \ldots, \gamma$,

$$
\widehat{g}_{i, x}(t) \delta x(t)+\widehat{g}_{i, u}(t) \delta u(t) \leq 0 \quad \text { whenever } \quad \widehat{g}_{i}(t)=0,
$$

and there exists a constant $M>0$ such that, for a.e. $t \in[0,1]$,

$$
\begin{aligned}
& \frac{\left[\widehat{g}_{i, x}(t) \delta x(t)+\widehat{g}_{i, u}(t) \delta u(t)\right]^{2}}{-\widehat{g}_{i}(t)} \leq M \\
& \text { whenever } \quad \widehat{g}_{i}(t)<0, \quad \widehat{g}_{i, x}(t) \delta x(t)+\widehat{g}_{i, u}(t) \delta u(t)>0 .
\end{aligned}
$$

$\left(\widetilde{C}_{7}\right)$ For all $i=1, \ldots, \kappa$,

$$
\widehat{k}_{i, x}(t) \delta x(t) \leq 0 \quad \text { whenever } \quad \widehat{k}_{i}(t)=0,
$$

and there exists a constant $M>0$ such that, for all $t \in[0,1]$,

$$
\frac{\left[\widehat{k}_{i, x}(t) \delta x(t)\right]^{2}}{-\widehat{k}_{i}(t)} \leq M \quad \text { whenever } \quad \widehat{k}_{i}(t)<0, \quad \widehat{k}_{i, x}(t) \delta x(t)>0 .
$$


Using these new conditions, the statement of Theorem 4.1 simplifies to the following result (cf. [PZ94b, Theorem 5]).

COROLlaRY 4.1. Let $(\widehat{x}, \widehat{u})$ be a regular weak local minimum for problem $(\widetilde{\mathrm{CP}})$. Then, for every regular critical arc $(\delta x, \delta u)$, there exist constants $\lambda \in \mathbb{R}, \alpha=$ $\left(\alpha_{1}, \ldots, \alpha_{r}\right) \in \mathbb{R}^{r}$, and $\beta=\left(\beta_{1}, \ldots, \beta_{s}\right) \in \mathbb{R}^{s}$, an absolutely continuous function $p:[0,1] \rightarrow \mathbb{R}^{n}$, two integrable functions $\varphi:[0,1] \rightarrow \mathbb{R}^{\gamma}$ and $\psi:[0,1] \rightarrow \mathbb{R}^{\delta}$, and a Borel regular vector-valued measure $\mu=\left(\mu_{1}, \ldots, \mu_{\kappa}\right)$, not all zero, such that $\lambda \geq 0$,

$$
\begin{gathered}
\alpha \geq 0, \quad \alpha^{T} \widehat{a}=0, \\
\varphi(t) \geq 0, \quad \varphi^{T}(t) \widehat{g}(t)=0 \quad \text { for a.e. } t \in[0,1], \\
\mu \geq 0, \quad \int_{0}^{1} \widehat{k}(t) d \mu(t)=0,
\end{gathered}
$$

(4.10), (4.11), (4.12), (4.13) hold, and

$$
\begin{aligned}
& \left(\lambda \widehat{\ell}^{\prime \prime}+\alpha^{T} \widehat{a}^{\prime \prime}+\beta^{T} \widehat{b}^{\prime \prime}\right)(\delta x(0), \delta x(1))+\int_{0}^{1} \widehat{k}^{\prime \prime}(t ; \delta x(t)) d \mu(t) \\
& +\int_{0}^{1} \widehat{\mathcal{H}}^{\prime \prime}\left(t, p(t)+\int_{] t, 1]} \widehat{k}_{x}^{T}(t) d \mu(t), \varphi(t), \psi(t) ; \delta x(t), \delta u(t)\right) d t \geq \sum_{i=1}^{\kappa} 2 \int_{0}^{1} \sigma_{\widehat{k}_{i}, \widehat{k}_{i x} \delta x}(t) d \mu_{i}(t),
\end{aligned}
$$

where $\sigma_{a, b}$ is defined by (2.14).

Remark 4.2. In the very special case when $g(t, x, u)=u$, the first-order necessary conditions in the above corollary form a special case of [Cla83, Theorem 5.2.1] and [Gir72]. On the other hand, when no state constraints are present, the first-order part of this corollary generalizes the results in [MOS98] and [OS95]. When only equality control constraints are present, the statement of Corollary 4.1 has its exact parallel in [ZZ88] for the case where the state is piecewise smooth and the control is piecewise continuous.

Proof. Define $R, Q$, and $S$ by (4.28). Using the product rule and Theorem 2.2, it follows that $\left(\widetilde{R}_{3}\right)$ implies $\left(R_{3}\right)$. Similarly, due to the product rule and Theorems 2.2 and 2.1 , it follows that conditions $\left(\widetilde{C}_{2}\right),\left(\widetilde{C}_{5}\right)$, and $\left(\widetilde{C}_{7}\right)$ are equivalent to $\left(C_{2}\right),\left(C_{5}\right)$, and $\left(C_{7}\right)$, respectively. Thus, all the assumptions of Theorem 4.1 are satisfied, and hence we also have its conclusions.

We can see that (4.7), (4.8), and (4.9) are equivalent to (4.36), (4.37), and (4.38), respectively. By the second part of Corollary 2.1, the first and third terms on the right-hand side of (4.14) vanish. By Corollary 2.2 the second term of (4.14) reduces to the right-hand side of (2.15). Therefore, (4.14) reduces to (4.39).

5. Example. Consider the problem

$$
\begin{array}{ll}
\text { Minimize } & x_{3}(1) \\
\text { subject to } & \left\{\begin{array}{l}
\dot{x_{1}}(t)=u_{1}(t) \text { for a.e. } t \in[-1,1] \\
\dot{x_{2}}(t)=u_{2}(t) \text { for a.e. } t \in[-1,1] \\
\dot{x_{3}}(t)=x_{1}^{3}(t)+\zeta(t) u_{2}(t) \text { for a.e. } t \in[-1,1] \\
x_{1}(-1)=x_{2}(-1)=x_{3}(-1)=0, \\
x_{1}(1)=x_{2}(1)=0, \\
-x_{2}(t)-\left(x_{1}(t)-t\right)^{2}-x_{1}^{2}(t) \leq 0 \text { for } t \in[-1,1]
\end{array}\right.
\end{array}
$$


where

$$
\zeta(t):= \begin{cases}0 & \text { if } t \in[-1,0), \\ -1 & \text { if } t \in[0,1] .\end{cases}
$$

The Hamiltonian of this problem is

$$
\mathcal{H}(t, x, u, p):=p_{1} u_{1}+p_{2} u_{2}+p_{3}\left(x_{1}^{3}+\zeta(t) u_{2}\right) .
$$

This problem is a special case of ( $(\widetilde{\mathrm{CP}})$, where (i), (iv), and (v) are absent and, for $x^{T}=\left(x_{1}, x_{2}, x_{3}\right)$ and $u^{T}=\left(u_{1}, u_{2}\right)$, we have

$$
\begin{gathered}
b^{T}(x(-1), x(1))=\left(x^{T}(-1), x_{1}(1), x_{2}(1)\right), \quad f^{T}(t, x, u)=\left(u_{1}, u_{2}, x_{1}^{3}+\zeta(t) u_{2}\right), \\
k(t, x)=-x_{2}-\left(x_{1}-t\right)^{2}-x_{1}^{2} .
\end{gathered}
$$

One would like to find out whether the admissible pair $(\widehat{x} ; \widehat{u})^{T}=(0,0,0 ; 0,0)$ is a good candidate for weak local minimality in $(\mathcal{C})$. For this reason, we shall check whether the first- and second-order necessary conditions presented by Corollary 4.1 hold true for this candidate.

We have $\widehat{k}(t)=-t^{2}$ and $\widehat{k}_{x}(t)=(2 t,-1,0)$. Now set

$$
\left.\lambda:=1, \quad p^{T}(t): \equiv(0,1,1), \quad \mu:=\delta_{0} \text { (the Dirac measure concentrated at } 0\right) .
$$

Then, by replacing the left endpoint 0 of the base interval in Theorem 4.1 and Corollary 4.1 by -1 , one can check that these multipliers (that are not all zero) uniquely (up to a nonzero constant multiple) satisfy (4.10), (4.13), and (4.38). By choosing the multipliers $\left(\beta_{1}, \ldots, \beta_{5}\right)$ (that correspond to the endpoint conditions) properly, (4.11) and (4.12) can also be satisfied.

Define, for $t \in[-1,1]$,

$$
\begin{aligned}
\delta x^{T}(t) & =\left(\delta x_{1}(t), \delta x_{2}(t), \delta x_{3}(t)\right):=(1-|t|, 0,0) \\
\text { and } \quad \delta u^{T}(t) & =\left(\delta u_{1}(t), \delta u_{2}(t)\right):=(-\operatorname{sign}(t), 0) .
\end{aligned}
$$

It follows that this choice of $\delta x$ satisfies (4.34) and, for $M=4$, (4.35). One can also check that all the remaining criticality conditions (and regularity conditions) are also satisfied. Therefore, $(\delta x, \delta u)$ is a regular critical direction for problem $(\mathcal{C})$.

It remains to check the inequality (4.39). Note that the first and the third terms there vanish. Using the definition of $\sigma_{\widehat{k}, \widehat{k}_{x} \delta x}$ in (2.14), inequality (4.39) simplifies to

$$
-4\left[\delta x_{1}(0)\right]^{2} \geq-2\left[\delta x_{1}(0)\right]^{2}
$$

which fails to hold, since $\delta x_{1}(0)=1$. Therefore, given that the conclusion of Corollary 4.1 is not valid, the pair $(\widehat{x} ; \widehat{u})^{T}=(0,0,0 ; 0,0)$ is not a weak local minimum for (e).

\section{REFERENCES}

[BT80] A. Ben-TAL, Second order theory of extremum problems, in Extremal Methods and System Analysis, A. V. Fiacco and K. Kortanek, eds., Springer-Verlag, Berlin, 1980, pp. 336-356. 
[BTZ82] A. BEn-TAL AND J. Zowe, A unified theory of first and second-order conditions for extremum problems in topological vector spaces, Math. Programming Stud., 19 (1982), pp. 39-76.

[Cla83] F. H. Clarke, Optimization and Nonsmooth Analysis, Canad. Math. Soc. Ser. Monogr. Adv. Texts, John Wiley, New York, 1983.

[Com90] R. CominetTi, Metric regularity, tangent sets, and second-order optimality conditions, Appl. Math. Optim., 21 (1990), pp. 265-287.

[DM63] A. YA. Dubovitskil And A. A. Milyutin, Extremum problems with constraints, Soviet Math. Dokl., 4 (1963), pp. 452-455.

[DM65] A. YA. Dubovitskil AND A. A. Milyutin, Second variations in extremal problems with constraints, Dokl. Akad. Nauk SSSR, 160 (1965), pp. 18-21.

[Gir72] I. V. GiRsanov, Lectures on Mathematical Theory of Extremum Problems, Lecture Notes in Econom. and Math. Systems 67, Springer-Verlag, Berlin, 1972.

[IT79] A. D. Ioffe And V. M. Tinomirov, Theory of Extremal Problems, North-Holland, Amsterdam, 1979.

[Kaw88a] H. KAWASAKI, An envelope like effect of infinitely many inequality constraints on secondorder necessary conditions for minimization problems, Math. Programming, 41 (1988), pp. 73-96.

[Kaw88b] H. KAWASAKI, The upper and second-order directional derivatives for a sup-type function, Math. Programming, 41 (1988), pp. 327-339.

[Kaw91] H. KAWASAKI, Second order necessary optimality conditions for minimizing a sup type function, Math. Programming, 49 (1991), pp. 213-229.

[Kaw92] H. KAWASAKI, Second order necessary and sufficient optimality conditions for minimizing a sup type function, Appl. Math. Optim., 26 (1992), pp. 195-220.

[MZ79] H. MAURER AND J. Zowe, First and second order necessary and sufficient conditions for infinite dimensional programming problems, Math. Programming, 16 (1979), pp. $98-110$.

[MOS98] A. A. Milyutin And N. P. Osmolovskir, Calculus of Variations and Optimal Control, Transl. Math. Monogr. 180, AMS, Providence, RI, 1998.

[OS95] N. P. Osmolovskil, Quadratic conditions for nonsingular extremals in optimal control (A theoretical treatment), Russ. J. Math. Phys., 2 (1995), pp. 487-516.

[PZ94a] Zs. PÁles AND V. ZEIDAN, Nonsmooth optimum problems with constraints, SIAM J. Control Optim., 32 (1994), pp. 1476-1502.

[PZ94b] Zs. PÁles AND V. ZeIdAn, First- and second-order necessary conditions for control problems with constraints, Trans. Amer. Math. Soc., 346 (1994), pp. 421-453.

[PZ96] Zs. PÁLES AND V. ZEIDAN, Second-order necessary conditions for nonsmooth optimum problems with constraints, in World Congress of Nonlinear Analysts '92, Vols. 1-4, de Gruyter, Berlin, 1996, pp. 2337-2346.

[PZ98] Zs. PÁLES AND V. ZEIDAN, Optimum problems with certain lower semicontinuous setvalued constraints, SIAM J. Optim., 8 (1998), pp. 707-727.

[PZ99a] Zs. PÁlES AND V. ZEIDAN, Characterization of closed and open C-convex sets in $\mathcal{C}\left(T, \mathbb{R}^{r}\right)$, Acta Sci. Math. (Szeged), 65 (1999), pp. 339-357.

[PZ99b] Zs. PÁLES AND V. ZEIDAN, On $L^{1}$-closed decomposable sets in $L_{\infty}$, in Systems Modelling and Optimization (Detroit, MI, 1997), Chapman \& Hall/CRC, Boca Raton, FL, 1999, pp. 198-206.

[PZ99c] Zs. PÁles And V. Zeidan, Characterization of $L^{1}$-closed decomposable sets in $L^{\infty}, \mathrm{J}$. Math. Anal. Appl., 238 (1999), pp. 491-515.

[PZ00] Zs. PÁLES AND V. ZEIDAN, Optimum problems with measurable set-valued constraints, SIAM J. Optim., 11 (2000), pp. 426-443.

[PZ01] Zs. PÁles AND V. ZEIDAN, The critical tangent cone in second-order conditions for optimal control, in Proceedings of the 2nd World Congress of Nonlinear Analysts, Catania, Italy, 2000, V. Lakshmikantham, ed., Nonlinear Anal., 47 (2001), pp. 11491161.

[SZ] G. Stefani And P. Zezza, Optimal control problems with mixed state-control constraints: Necessary conditions, J. Math. Systems Estim. Control, 2 (1992), pp. 155189.

[ZZ88] V. ZEIDAN AND P. ZEZZA, The conjugate point condition for smooth control sets, J. Math. Anal. Appl., 132 (1988), pp. 572-589. 